\title{
ZERO VISCOSITY AND THERMAL DIFFUSIVITY LIMIT OF THE LINEARIZED COMPRESSIBLE NAVIER-STOKES-FOURIER EQUATIONS IN THE HALF PLANE
}

\author{
YUTAO DING AND NING JIANG
}

\begin{abstract}
We study the zero viscosity and heat conductivity limit of an initial boundary problem for the linearized Navier-Stokes-Fourier equations of a compressible viscous and heat conducting fluid in the half plane. We consider the case that the viscosity and thermal diffusivity converge to zero at the same order. The approximate solution of the linearized NavierStokes-Fourier equations with inner and boundary expansion terms is analyzed formally first by multiscale analysis. Then the pointwise estimates of the error terms of the approximate solution are obtained by energy methods, thus establish the uniform stability for the linearized Navier-Stokes-Fourier equations in the zero viscosity and heat conductivity limit. This work is based on 8 and generalize the results from isentropic case to the general compressible fluid with thermal diffusive effect. Besides the viscous layer as in [8], thermal layer appears and coupled with the viscous layer linearly.
\end{abstract}

\section{INTRODUCTION}

The evolution of a compressible viscous heat conducting fluid occupying in the half-plane can be described by the density $\rho(t, x) \geq 0$, the velocity $\mathrm{u}(t, x) \in \mathbb{R}^{2}$, and the temperature $\theta(t, x) \geq 0$ obeying the following Navier-Stokes-Fourier system of equations:

$$
\begin{aligned}
\partial_{t} \rho+\nabla_{x} \cdot(\rho \mathrm{u}) & =0 \\
\partial_{t}(\rho \mathrm{u})+\nabla_{x} \cdot(\rho \mathrm{u} \otimes \mathrm{u})+\nabla_{x} p & =\nabla_{x} \cdot \mathbb{S}, \\
\partial_{t}(\rho Q(\theta))+\nabla_{x} \cdot(\rho Q(\theta) u)+\theta p_{\theta} \nabla_{x} \cdot \mathrm{u} & =\mathbb{S}: \nabla_{x} u-\nabla_{x} \cdot q,
\end{aligned}
$$

with $(t, x) \in \mathbb{R}_{+} \times \Omega$. Here $\Omega=\left\{\left(x_{1}, x_{2}\right) \in \mathbb{R}^{2}, x_{1}>0\right\}$ with boundary $\Gamma=\partial \Omega=\left\{\left(x_{1}, x_{2}\right) \in\right.$ $\left.\mathbb{R}^{2}, x_{1}=0\right\}$. In the system (1.1),$Q \in C^{2}[0, \infty)$ is a given function such that

$$
Q(\theta)=\int_{0}^{\theta} c_{v}(z) \mathrm{d} z, \quad c_{v}(z) \geq c_{v}>0 \text { for } z>0 .
$$

The quantities $p, \mathbb{S}$, and $q$ are determined in terms of $\rho, \nabla_{x} \mathrm{u}$ and $\theta$ through constitutive equations: the first, Newton's law of viscosity, $\mathbb{S}(\mathrm{u})=\mu\left(\nabla_{x} \mathrm{u}+\nabla_{x} \mathrm{u}^{\top}\right)+\lambda \nabla_{x} \cdot \mathrm{uII}$, with constant viscosity coefficients $\mu, \lambda$ satisfying $\mu>0, \xi=\lambda+\mu \geq 0$. The second, the state equation $p=p_{e}(\rho)+$ $\theta p_{\theta}(\rho)$. The third, Fourier's law $q=-\kappa \nabla_{x} \theta$ with the thermal diffusivity $\kappa>0$. More details of the derivation of the system (1.1) can be found in [1].

In this paper, we study the system (1.1) imposed with the non-slip boundary condition for any $T>0$,

$$
\mathrm{u}=0, \quad \theta=0 \quad \text { on } \quad \Gamma \times[0, T]
$$

and the initial data

$$
\left(p, \mathrm{u}_{1}, \mathrm{u}_{2}, \theta\right)^{\top}(x, 0)=\left(p_{0}, \mathrm{u}_{1,0}, \mathrm{u}_{2,0}, \theta_{0}\right)^{\top}(x), \quad x \in \Omega .
$$

On the other hand, the motion of an inviscid compressible fluid without thermal diffusivity is governed by the compressible Euler equations, which are obtained by formally taking the 
viscosity coefficients $\mu, \lambda$ and the thermal diffusivity $\kappa$ as zeros in (1.1).

$$
\begin{aligned}
\partial_{t} \rho+\nabla_{x} \cdot(\rho \mathrm{u}) & =0, \\
\partial_{t}(\rho \mathrm{u})+\nabla_{x} \cdot(\rho \mathrm{u} \otimes \mathrm{u})+\nabla_{x} p & =0, \\
\partial_{t}(\rho Q(\theta))+\nabla_{x} \cdot(\rho Q(\theta) u)+\theta p_{\theta} \nabla_{x} \cdot \mathrm{u} & =0 .
\end{aligned}
$$

The boundary condition imposed on the compressible Euler equations (1.4) is

$$
\mathrm{u}_{1}=0 \quad \text { on } \Gamma \times[0, T] .
$$

We impose the same initial data for (1.4) as in (1.1), i.e. (1.3).

In [8, Xin and Yanagisawa studied the zero viscosity limit of the linearized Navier-Stokes equations for an isentropic compressible viscous fluid in the half plane. In other words, they considered the equations (1.1) without the energy equation, and the pressure $p=p(\rho)$ depending only on the density $\rho$. Under the assumption that the coefficients of viscosity $\mu$ and the bulk viscosity $\xi=\mu+\lambda$ have the same order $\varepsilon^{2}$, they investigated the asymptotic behavior of the solution of an Dirichlet boundary value problem of the linearized Navier-Stokes equation as the parameter $\varepsilon$ goes to zero. It is well-known that due to the disparity of the boundary conditions between Navier-Stokes and Euler equations, a thin region, the so-called boundary layer comes out near the boundary $\Gamma$ in which the values of the unknown functions change drastically in this zero viscosity limit.

In [8], by clarifying the special structure of the boundary matrix of the Euler part of the linearized Navier-Stokes equations, Xin and Yanagisawa introduced the boundary characteristic variables and used the asymptotic analysis with multiple length scales to construct an approximate solution of the initial boundary value problem of the linearized Navier-Stokes equations, which included the inner and boundary layer terms. The first order term of the inner expansion is determined by the solution of the linearized Euler equations, i.e. acoustic system, while the the terms in the boundary expansion are solutions of a family of ODEs and Prandtl-type equations. Next, they used the energy method to show the pointwise error estimates of the approximate solution with respect to the viscosity, and derived the uniform stability results of the linearized Navier-Stokes solutions in the zero-viscosity limit.

The present work could be considered as a follow up of [8]. We also only study the linearized problem of the Navier-Stokes-Fourier equations for a compressible viscous fluid with thermal diffusivity. The major difficulty in the research of the boundary layers of the original nonlinear problem lies in the fact that the leading boundary layer terms satisfy the nonlinear Prandtl-type equations, for which even the local in time existence and regularity in usual Sobolev spaces are wide open problems so far. The only available zero-viscosity limit result for analytic solutions of the nonlinear incompressible Navier-Stokes equations in half-space is due to Sammartino and Caflisch [4, [5]. The analogue of the same type results for the compressible Navier-Stokes equations is not known.

The purpose of the current paper aims to generalize Xin-Yanagisawa's result by adding the energy (or equivalently temperature) equation. We make the assumption that the thermal diffusivity $\kappa$ is proportional to $\varepsilon^{2}$, the same order with viscosities. This assumption is for the simplicity of analyzing the structure of viscosity and thermal boundary layers. Physically, the viscosity and thermal diffusivity could have different order. For example, the viscosity is of order $\varepsilon^{2}$ while the thermal diffusivity is of order $\varepsilon^{\gamma}$ for some $\gamma>0$. For this more physical case, even at the formal level, the viscous and thermal layers are not clear so far.

We would like to remark that the case that the viscosity and thermal diffusivity have the same order $\varepsilon^{2}$ is physically meaningful and interesting. If we start from mesoscopic level of gas dynamics, say, the Boltzmann equation and set the Knudsen number as $\varepsilon^{2}$. It can be derived formally under the assumption that the deviation from the global Maxwellian with size much 
smaller than the Knudsen number, the leading fluid dynamics is exactly the linearized NavierStokes-Fourier equations for ideal gas with the viscosity and thermal diffusivity of the same order $\varepsilon^{2}$. In the work under preparation by the second author and N. Masmoudi [2], the acoustic dynamics of the linearized Boltzmann equation with Maxwell reflection boundary condition in half space is studied. The limiting process considered there includes the zero viscosity and thermal diffusivity limit in the current paper (for ideal gas).

For the linearized Euler equations, i.e. the acoustic system, the boundary condition is (1.5), and no boundary condition for $\theta$. The disparity between the boundary conditions for the linearized Navier-Stokes-Fourier equations, (1.2) and the condition (1.5) suggest that during the limit $\varepsilon \rightarrow 0$, both viscous and thermal boundary layers are generated.

In this paper, we employ the strategy used in [8]. We first construct an approximate solutions of the linearized Navier-Stokes-Fourier equations with non-slip boundary condition which includes inner and boundary layer terms. Then using the energy method, we established the pointwise estimates for the error terms, thus derive the uniform stability results for the linearized Navier-Stokes-Fourier solutions in the zero viscosity and thermal diffusivity limits. Our result in this paper is a preliminary consideration of the coupling thermal and viscous layers of the a compressible fluid with both viscosity and thermal diffusivity effects.

Comparing with [8], the main novelty of this work is the appearance of the thermal layer. The key point is that because the viscosity and the thermal diffusivity have the same order, the viscous layer and thermal layer appear also appear at the same order, and more importantly, these two types of layers are coupled linearly. Technically, this fact is reflected by that in [8], the viscous layer was described by a single Prandtl-type equation, while in the current work, the viscous and thermal layers are described by a linear system of two Prandtl-type equations. More importantly, the coupling of viscous and thermal layers are linear and weak in the sense that the coupling of the system is only on the unknown functions themselves, but not on their derivatives. This fact also make the analysis easier. We believe that for the case that the viscosity if of order $\varepsilon^{2}$, while the thermal diffusivity is of order $\varepsilon^{\gamma}$ with $0<\gamma \neq 2$, these two layers will strongly coupled together. We plan to study this interesting and challenging problem in the near future.

The organization of the paper is as follows: In the rest of this section, we introduce the setting of the problem and state the main theorem. In Section 3, using the method of multiple scales, the approximate solution of initial boundary problem of the linearized Navier-Stokes-Fourier equations (1.7) is constructed. Section 3 is devoted to the estimates of the error term by energy method. In the last section, we collect some know results and prove the existence of the linear system of Prandtl-type equation.

1.1. The Setting of the Problem and the Main Result. Now, we first set up the linearized problem of (1.1)-(1.3). Let $V=\left(\rho, \mathrm{u}_{1}, \mathrm{u}_{2}, \theta\right)^{\top}$, we rewrite the (1.1) as the following symmetric form

$$
A_{0}(V) \partial_{t} V+\sum_{j=1}^{2} A_{j}(V) \partial_{j} V=L V
$$

where

$$
A_{0}(V)=\left(\begin{array}{cccc}
\frac{1}{\rho} & 0 & 0 & 0 \\
0 & \frac{\rho}{p_{\rho}} & 0 & 0 \\
0 & 0 & \frac{\rho}{p_{\rho}} & 0 \\
0 & 0 & 0 & \beta
\end{array}\right), \quad A_{1}(V)=\left(\begin{array}{cccc}
\frac{\mathrm{u}_{1}}{\rho} & 1 & 0 & 0 \\
1 & \frac{\rho \mathrm{u}_{1}}{p_{\rho}} & 0 & \frac{p_{\theta}}{p_{\rho}} \\
0 & 0 & \frac{\rho \mathrm{u}_{1}}{p_{\rho}} & 0 \\
0 & \frac{p_{\theta}}{p_{\rho}} & 0 & \beta \mathrm{u}_{1}
\end{array}\right)
$$


and

$$
A_{2}(V)=\left(\begin{array}{cccc}
\frac{\mathrm{u}_{2}}{\rho} & 0 & 1 & 0 \\
0 & \frac{\rho \mathrm{u}_{2}}{p_{\rho}} & 0 & 0 \\
1 & 0 & \frac{\rho \mathrm{u}_{2}}{p_{\rho}} & \frac{p_{\theta}}{p_{\rho}} \\
0 & 0 & \frac{p_{\theta}}{p_{\rho}} & \beta \mathrm{u}_{2}
\end{array}\right), \quad L V=\left(\begin{array}{c}
0 \\
\frac{\mu}{p_{\rho}} \Delta \mathrm{u}_{1}+\frac{\xi}{p_{\rho}}\left(\partial_{11} \mathrm{u}_{1}+\partial_{12} \mathrm{u}_{2}\right) \\
\frac{\mu}{p_{\rho}} \Delta \mathrm{u}_{2}+\frac{\xi}{p_{\rho}}\left(\partial_{21} \mathrm{u}_{1}+\partial_{22} \mathrm{u}_{2}\right) \\
\left.\frac{\kappa}{\theta p_{\rho}} \Delta \theta+\frac{1}{\theta p_{\rho}} \mathbb{S}: \nabla_{x} \mathrm{u}\right)
\end{array}\right)
$$

where $\beta=\frac{\rho c_{v}(\theta)}{\theta p_{\rho}}, p_{\rho}, p_{\theta}$ denote the partial derivatives $\frac{\partial p}{\partial \rho}, \frac{\partial p}{\partial \theta}$, and $\partial_{i j}$ denotes $\partial_{x_{i} x_{j}}$.

Let $\mu=\bar{\mu} \varepsilon^{2}, \xi=\bar{\xi} \varepsilon^{2}=(\bar{\mu}+\bar{\lambda}) \varepsilon^{2}$ and $\kappa=\bar{\kappa} \varepsilon^{2}$ where $\varepsilon$ is a small positive parameter, the constants $\bar{\mu}, \bar{\lambda}, \bar{\kappa}$ are of order 1 and independent of $\varepsilon$. We linearize equations (1.1) around smooth functions $V^{\prime}(t)=\left(\rho^{\prime}, \mathrm{u}_{1}^{\prime}, \mathrm{u}_{2}^{\prime}, \theta^{\prime}\right)$ which is a solution to the equations (1.1) for $t \in[0, T]$ for some $T>0$. Then the linearized equations of (1.1) can be written as equations for $V^{\varepsilon}=$ $\left(\rho^{\varepsilon}, v_{1}^{\varepsilon}, v_{2}^{\varepsilon}, \theta^{\varepsilon}\right)^{\top}$ :

$$
\begin{aligned}
A_{0}\left(V^{\prime}\right) \partial_{t} V^{\varepsilon}+\sum_{j=1}^{2} A_{j}\left(V^{\prime}\right) \partial_{j} V^{\varepsilon} & =D_{\varepsilon} V^{\varepsilon} \quad \text { in } \quad \Omega \times[0, T], \\
M^{+} V^{\varepsilon} & =0 \quad \text { on } \quad \Gamma \times[0, T], \\
V^{\varepsilon}(x, 0) & =\left(\rho_{0}, v_{1,0}, v_{2,0}, \theta_{0}\right)^{\top}(x)=V_{0}(x) \quad \text { for } \quad x \in \Omega,
\end{aligned}
$$

where

$$
D_{\varepsilon} V=\varepsilon^{2}\left(\begin{array}{c}
0 \\
\frac{\bar{\mu}}{p_{\rho}^{\prime}} \Delta v_{1}+\frac{\bar{\xi}}{p_{\rho}^{\prime}}\left(\partial_{11} v_{1}+\partial_{12} v_{2}\right) \\
\frac{\bar{\xi}}{p_{\rho}^{\prime}} \Delta v_{2}+\frac{\bar{\xi}}{p_{\rho}^{\prime}}\left(\partial_{21} v_{1}+\partial_{22} v_{2}\right) \\
\frac{\bar{\kappa}}{\theta^{\prime} p_{\rho}^{\prime}} \Delta \theta+I(V)
\end{array}\right), \quad M^{+}=\left(\begin{array}{cccc}
0 & 1 & 0 & 0 \\
0 & 0 & 1 & 0 \\
0 & 0 & 0 & 1
\end{array}\right)
$$

and

$$
I(V)=\frac{2 \bar{\mu}}{\theta^{\prime} p_{\rho}^{\prime}}\left(\partial_{i} \mathrm{u}_{j}^{\prime}+\partial_{j} \mathrm{u}_{i}^{\prime}\right) \partial_{i} v_{j}+\frac{2 \bar{\lambda}}{\theta^{\prime} p_{\rho}^{\prime}}\left(\operatorname{div} u^{\prime}\right)(\operatorname{div} v) .
$$

We can rewrite $D_{\varepsilon} V$ as matrices form:

$$
D_{\varepsilon} V=\varepsilon^{2}\left(\begin{array}{cccc}
0 & 0 & 0 & 0 \\
0 & \frac{\bar{\mu}}{p_{\rho}^{\prime}} & 0 & 0 \\
0 & 0 & \frac{\bar{\mu}}{p_{\rho}^{\prime}} & 0 \\
0 & 0 & 0 & \frac{\bar{\kappa}}{\theta^{\prime} p_{\rho}^{\prime}}
\end{array}\right) \Delta V+\varepsilon^{2} \frac{\bar{\xi}}{p_{\rho}^{\prime}}\left(\begin{array}{cccc}
0 & 0 & 0 & 0 \\
0 & \partial_{11} & \partial_{12} & 0 \\
0 & \partial_{21} & \partial_{22} & 0 \\
0 & 0 & 0 & 0
\end{array}\right) V+\varepsilon^{2} \sum_{j=1}^{2} I_{j} \partial_{j} V
$$

where

$$
I_{1}=\left(\begin{array}{cccc}
0 & 0 & 0 & 0 \\
0 & 0 & 0 & 0 \\
0 & 0 & 0 & 0 \\
0 & 2 \frac{\bar{\mu}}{\theta^{\prime} p_{\rho}^{\prime}} \partial_{1} \mathrm{u}_{1}^{\prime}+\frac{\bar{\lambda}}{\theta^{\prime} p_{\rho}^{\prime}} \operatorname{divu} \mathrm{u}^{\prime} & \frac{\bar{\mu}}{\theta^{\prime} p_{\rho}^{\prime}}\left(\partial_{1} \mathrm{u}_{2}^{\prime}+\partial_{2} \mathrm{u}_{1}^{\prime}\right) & 0
\end{array}\right)
$$

and

$$
I_{2}=\left(\begin{array}{cccc}
0 & 0 & 0 & 0 \\
0 & 0 & 0 & 0 \\
0 & 0 & 0 & 0 \\
0 & \frac{\bar{\mu}}{\theta^{\prime} p_{\rho}^{\prime}}\left(\partial_{1} \mathrm{u}_{2}^{\prime}+\partial_{2} \mathrm{u}_{1}^{\prime}\right) & 2 \frac{\bar{\mu}}{\theta^{\prime} p_{\rho}^{\prime}} \partial_{2} \mathrm{u}_{2}^{\prime}+\frac{\bar{\lambda}}{\theta^{\prime} p_{\rho}^{\prime}} \operatorname{divu}^{\prime} & 0
\end{array}\right)
$$


The corresponding initial boundary value problems of the linearized Euler equation are

$$
\begin{aligned}
A_{0}\left(V^{\prime}\right) \partial_{t} V^{0}+\sum_{j=1}^{2} A_{j}\left(V^{\prime}\right) \partial_{j} V^{0} & =0 \quad \text { in } \quad \Omega \times[0, T], \\
M^{0} V^{0} & =0 \quad \text { on } \quad \Gamma \times[0, T], \\
V^{0}(x, 0) & =V_{0}(x) \quad \text { for } \quad x \in \Omega,
\end{aligned}
$$

where $M^{0}=(0,1,0,0)$.

Before we state our results, we introduce some function spaces and the notion of compatibility condition. Let $m \geq 0$ be an integer and $U \subset \mathbb{R}^{d}, d \geq 1$ be a domain. Then $H^{m}(U)$ denotes the usual Sobolev space of order $m$ equipped with the norm $\|\cdot\|_{m, U}$ and the inner product $(\cdot, \cdot)_{m, U}$. For $0<\alpha<1, C^{\alpha}(U)$ denotes the Hölder space on $\bar{U}$ with the exponent $\alpha$, endowed with the norm $\|\cdot\|_{C^{\alpha}(U)}$. The space $C^{m}(I ; X)$ denotes the set of functions $u(t), t \in I$, the $m$-times continuously differentiable functions on the interval $I$ with value taken in the Banach space $X$.

To study the initial boundary value problem (1.7), we need the following compatibility condition: Define inductively the n-Cauchy data of (1.7) by

$$
\begin{aligned}
\dot{\partial}_{t}^{0} V^{\varepsilon}(x, 0)= & V^{\text {in }}(x), \\
\dot{\partial}_{t}^{n} V^{\varepsilon}(x, 0)= & \sum_{s=0}^{n-1} C_{n-1}^{s}\left\{-\sum_{j=1}^{2} \partial_{t}^{s}\left(A_{0}^{-1}\left(V^{\prime}\right) A_{j}\left(V^{\prime}\right)\right)(x, 0) \dot{\partial}_{t}^{n-1-s} V^{\varepsilon}(x, 0)\right. \\
& \left.+\partial_{t}^{s} A_{0}^{-1}\left(V^{\prime}\right) \dot{\partial}_{t}^{n-1-s} B\left(\varepsilon^{2}, c_{1} \varepsilon^{2}, c_{2} \varepsilon^{2}\right) V^{\varepsilon}(x, 0)\right\} \quad \text { for } \quad n=1,2, \cdots
\end{aligned}
$$

The initial data $V^{\text {in }}(x)$ is said to satisfy the compatibility condition of order $m$ for the initial boundary value problem (1.7) for any $\varepsilon>0$ if $M^{+} \dot{\partial}_{t}^{n} V^{\varepsilon}(x, 0)=0$, i.e.

$$
\dot{\partial}_{t}^{n} v_{1}(x, 0)=\dot{\partial}_{t}^{n} v_{2}(x, 0)=\dot{\partial}_{t}^{n} \theta(x, 0) \quad \text { on } \quad \Gamma, \quad n=0,1, \cdots, m, \quad \text { for any } \varepsilon>0 .
$$

The condition (1.12) implies the corresponding compatibility condition of order $m$ of the initial boundary value problem for linearized Euler equation (1.10):

$$
M^{0} \dot{\partial}_{t}^{n} V^{0}(x, 0)=0 \quad \text { on } \quad \Gamma, \quad n=0,1, \cdots, m,
$$

where $\dot{\partial}_{t}^{n} V^{0}(x, 0)$ are defined by (1.11) in which $\varepsilon$ is taken as zero.

Then we state the main theorem of this paper

Theorem 1.1. Let $m$ be an integer satisfies $m \geq 2(7 N+4)$. Suppose that the initial data $V_{0} \in H^{m}(\Omega)$ satisfies the compatibility condition of order $\left[\frac{m}{2}\right]-1$ for (1.7) for any $\varepsilon>0$ and the compatibility condition of order $m-1$ for (1.10). Then the solution $V=\left(\rho, v_{1}, v_{2}, \theta\right)^{\top}$ of problem (1.7), the solution $E=\left(\rho^{E}, v_{1}^{E}, v_{2}^{E}, \theta^{E}\right)^{\top}$ of (1.10) and the correcting term $K^{\varepsilon}=U^{\varepsilon}-E, W^{\varepsilon}$ is defined in (2.29), exist uniquely in the following spaces:

$$
\begin{gathered}
V \in \bigcap_{j=0}^{[m / 2]} C^{j}\left([0, T] ; H^{m-2 j}(\Omega)\right), \quad E \in \bigcap_{j=0}^{m} C^{j}\left([0, T] ; H^{m-j}(\Omega)\right), \\
K^{\varepsilon} \in \bigcap_{j=0}^{[m / 2]-1-7 N} C^{j}\left([0, T] ; H^{[m / 2-1-7 N-j]}(\Omega)\right),
\end{gathered}
$$

and for $0<\varepsilon<1$, there exist constants $C_{1}$ and $C_{2}$ which are independent of $\varepsilon$, such that the following estimate hold:

$$
\sup _{(x, t) \in \Omega \times[0, T]}\left|\rho(x, t)-\rho^{E}(x, t)-K_{0}^{\varepsilon}(x, t)\right| \leq C_{1} \varepsilon^{N-1},
$$




$$
\sup _{(x, t) \in \Omega \times[0, T]}\left|v_{j}(x, t)-v_{j}^{E}(x, t)-K_{j}^{\varepsilon}(x, t)\right| \leq C_{2} \varepsilon^{N-3 / 4}, \quad j=1.2,
$$

and

$$
\sup _{(x, t) \in \Omega \times[0, T]}\left|\theta(x, t)-\theta^{E}(x, t)-K_{3}^{\varepsilon}(x, t)\right| \leq C_{2} \varepsilon^{N-3 / 4} .
$$

\section{Construction of an Approximate Solution}

Throughout this section, we denote the solution of (1.7) by $V$ instead of $V^{\varepsilon}$ for simplicity.

2.1. Boundary Characteristic Variables. In the isentropic case [8], the matrix $A_{1}\left(V^{\prime}\right)$ is diagonalized. In this paper, for the non-isentropic case, this diagonalization process is not easy. Instead, we decompose $A_{1}\left(V^{\prime}\right)$ into two parts: one is easy to be diagonalized, the other vanishes on the boundary $\Gamma$. Let $A_{1 m}=A_{1 m}\left(x_{2}, t\right)=A_{1}\left(V^{\prime}\left(0, x_{2}, t\right)\right)$, then

$$
\begin{aligned}
A_{1}\left(V^{\prime}\right)= & A_{1 m}\left(V^{\prime}\right)+A_{1 r}\left(V^{\prime}\right) \\
& =\left(\begin{array}{cccc}
0 & 1 & 0 & 0 \\
1 & 0 & 0 & \alpha \\
0 & 0 & 0 & 0 \\
0 & \alpha & 0 & 0
\end{array}\right)+\left(\begin{array}{cccc}
\frac{\mathrm{u}_{1}^{\prime}}{\rho^{\prime}} & 0 & 0 & 0 \\
0 & \frac{\rho^{\prime} \mathrm{u}_{1}^{\prime}}{p_{\rho}^{\prime}} & 0 & \frac{p_{\theta}^{\prime}}{p_{\rho}^{\prime}}-\alpha \\
0 & 0 & \frac{\rho^{\prime} \mathrm{u}_{1}^{\prime}}{p_{\rho}^{\prime}} & 0 \\
0 & \frac{p_{\theta}^{\prime}}{p_{\rho}^{\prime}}-\alpha & 0 & \beta^{\prime} \mathrm{u}_{1}^{\prime}
\end{array}\right)
\end{aligned}
$$

where $\alpha=\alpha\left(x_{2}, t\right)=\frac{p_{\theta}^{\prime}}{p_{\rho}^{\prime}}\left(0, x_{2}, t\right)$ is valued on the boundary $\Gamma=\left\{\left(0, x_{2}\right): x_{2} \in \mathbb{R}\right\}$. Note that the matrix $A_{1 r}$ vanishes on the boundary $\Gamma \times[0, T]$. In other words, $A_{1 r}$ does not contribute the nonzero eigenvalues and eigenvectors of $A_{1}$ on the boundary. In this sense, we call $A_{1 m}$ the main part of the matrix $A_{1}\left(V^{\prime}\right)$ of the hyperbolic part of the equation (1.7).

Simple calculations show that the eigenvalues of $A_{1 m}$ are $\lambda_{0}=\lambda_{1}=0, \lambda_{2}=\sqrt{\alpha^{2}+1}$, and $\lambda_{3}=-\sqrt{\alpha^{2}+1}$. Note that for the isentropic case $p_{\theta}=0$, so the eigenvalues are $0,1,-1$, which are reduced to the case considered in [8]. The corresponding right orthonormal eigenvector of $A_{1 m}$ are given by

$$
\begin{aligned}
& e_{0}=(0,0,1,0)^{\top}, \\
& e_{1}=\left(\frac{\alpha}{\sqrt{\alpha^{2}+1}}, 0,0, \frac{-1}{\sqrt{\alpha^{2}+1}}\right)^{\top}, \\
& e_{2}=\left(\frac{1}{\sqrt{2\left(\alpha^{2}+1\right)}}, \frac{1}{\sqrt{2}}, 0, \frac{\alpha}{\sqrt{2\left(\alpha^{2}+1\right)}}\right)^{\top}, \\
& e_{3}=\left(\frac{1}{\sqrt{2\left(\alpha^{2}+1\right)}},-\frac{1}{\sqrt{2}}, 0, \frac{\alpha}{\sqrt{2\left(\alpha^{2}+1\right)}}\right)^{\top} .
\end{aligned}
$$

Let $Q=\left(e_{0}, e_{1}, e_{2}, e_{3}\right)^{\top}$, then $Q$ is an orthogonal matrix, $Q^{-1}=Q^{\top}$. We define the boundary characteristic variables by

$$
U=\left(\begin{array}{l}
\mathrm{u}_{0} \\
\mathrm{u}_{1} \\
\mathrm{u}_{2} \\
\mathrm{u}_{3}
\end{array}\right)=Q V=\left(\begin{array}{c}
v_{2} \\
\frac{\rho-\theta}{\sqrt{\alpha^{2}+1}} \\
\frac{\rho-\alpha \theta}{\sqrt{2\left(\alpha^{2}+1\right)}}+\frac{v_{1}}{2} \\
\frac{\rho-\alpha \theta}{\sqrt{2\left(\alpha^{2}+1\right)}}-\frac{v_{1}}{2}
\end{array}\right),
$$

in terms of which the linearized Navier-Stokes-Fourier equations (1.7) can be transformed into

$$
\mathcal{A}_{0} \partial_{t} U-\mathcal{L}^{\varepsilon} U=0,
$$


where

$$
\begin{aligned}
\mathcal{L}^{\varepsilon}= & \sum_{j=1}^{2}\left\{-\mathcal{A}_{j}(x, t)+\varepsilon^{2} \mathcal{P}_{j}(x, t)+\varepsilon^{2} \mathcal{I}_{j}(x, t)\right\} \partial_{j} \\
& +\left(-\mathcal{W}(x, t)+\varepsilon^{2} \mathcal{Q}^{1}(x, t)+\varepsilon^{2} \mathcal{Q}^{2}(x, t)\right)+\varepsilon^{2} \mathcal{G}(x, t) \Delta+\varepsilon^{2} \bar{\xi} \sum_{i, j=1}^{2} \mathcal{G}^{i j} \partial_{i j}
\end{aligned}
$$

Here

$$
\begin{aligned}
& \mathcal{A}_{0}(x, t)=Q A_{0} Q^{-1}= \\
& \left(\begin{array}{cccc}
\frac{\rho^{\prime}}{p_{\rho}^{\prime}} & 0 & 0 & 0 \\
0 & \frac{1}{\alpha^{2}+1}\left(t \frac{\alpha^{2}}{\rho^{\prime}}+\beta^{\prime}\right) & \frac{1}{\alpha^{2}+1}\left(\frac{\alpha}{\sqrt{2} \rho^{\prime}}-\frac{\alpha \beta^{\prime}}{\sqrt{2}}\right) & \frac{1}{\alpha^{2}+1}\left(\frac{\alpha}{\sqrt{2} \rho^{\prime}}-\frac{\alpha \beta^{\prime}}{\sqrt{2}}\right) \\
0 & \frac{1}{\alpha^{2}+1}\left(\frac{\alpha}{\sqrt{2} \rho^{\prime}}-\frac{\alpha \beta^{\prime}}{\sqrt{2}}\right) & \frac{1}{\alpha^{2}+1}\left(\frac{1}{2 \rho^{\prime}}+\frac{\alpha^{2} \beta^{\prime}}{2}\right)+\frac{\rho^{\prime}}{2 p_{\rho}^{\prime}} & \frac{1}{\alpha^{2}+1}\left(\frac{1}{2 \rho^{\prime}}+\frac{\alpha^{2} \beta^{\prime}}{2}\right)-\frac{\rho^{\prime}}{2 p_{\rho}^{\prime}} \\
0 & \frac{1}{\alpha^{2}+1}\left(\frac{\alpha}{\sqrt{2} \rho^{\prime}}-\frac{\alpha \beta^{\prime}}{\sqrt{2}}\right) & \frac{1}{\alpha^{2}+1}\left(\frac{1}{2 \rho^{\prime}}+\frac{\alpha^{2} \beta^{\prime}}{2}\right)-\frac{\rho^{\prime}}{2 p_{\rho}^{\prime}} & \frac{1}{\alpha^{2}+1}\left(\frac{1}{2 \rho^{\prime}}+\frac{\alpha^{2} \beta^{\prime}}{2}\right)+\frac{\rho^{\rho}}{2 p_{\rho}^{\prime}}
\end{array}\right) \\
& =\left(\begin{array}{cccc}
\frac{\rho^{\prime}}{p_{\rho}^{\prime}} & 0 & 0 & 0 \\
0 & \eta_{0} & \eta_{1} & \eta_{1} \\
0 & \eta_{1} & \eta_{2} & \eta_{3} \\
0 & \eta_{1} & \eta_{3} & \eta_{2}
\end{array}\right) .
\end{aligned}
$$

and $\mathcal{A}_{1}(x, t)=\mathcal{A}_{1 m}+\mathcal{A}_{1 r}$,

$$
\begin{gathered}
\mathcal{A}_{1 m}=Q A_{1 m} Q^{-1}=\left(\begin{array}{cccc}
0 & 0 & 0 & 0 \\
0 & 0 & 0 & 0 \\
0 & 0 & \sqrt{\alpha^{2}+1} & 0 \\
0 & 0 & 0 & -\sqrt{\alpha^{2}+1}
\end{array}\right), \\
\mathcal{A}_{1 r}=Q A_{1 r} Q^{-1}, \quad \mathcal{A}_{2}=Q A_{2} Q^{-1} \\
\mathcal{P}_{j}(x, t)=2 Q\left(\begin{array}{cccc}
0 & 0 & 0 & 0 \\
0 & \frac{\bar{\mu}}{p_{\rho}^{\prime}} & 0 & 0 \\
0 & 0 & \frac{\bar{\mu}}{p_{\rho}^{\prime}} & 0 \\
0 & 0 & 0 & \frac{\bar{\kappa}}{\theta^{\prime} p_{\rho}^{\prime}}
\end{array}\right) \partial_{j} Q^{-1}, \quad \mathcal{I}_{j}(x, t)=Q I_{j} Q^{-1}, \\
\mathcal{W}(x, t)=Q A_{0} \partial_{t} Q^{-1}+\sum_{j=1}^{2} Q A_{j} \partial_{j} Q^{-1}, \\
\mathcal{Q}^{1}(x, t)=Q\left(\begin{array}{cccc}
0 & 0 & 0 & 0 \\
0 & \frac{1}{p_{\rho}^{\prime}} & 0 & 0 \\
0 & 0 & \frac{1}{p_{\rho}^{\prime}} & 0 \\
0 & 0 & 0 & \frac{\kappa_{0}\left(\theta^{\prime}\right)}{\theta^{\prime} p_{\rho}^{\prime}}
\end{array}\right) \Delta Q^{-1}, \quad \mathcal{Q}^{2}(x, t)=\sum_{j=1}^{2} Q I_{j} \partial_{j} Q^{-1},
\end{gathered}
$$




$$
\begin{aligned}
& \mathcal{G}(x, t)=Q\left(\begin{array}{cccc}
0 & 0 & 0 & 0 \\
0 & \frac{1}{p_{\rho}^{\prime}} & 0 & 0 \\
0 & 0 & \frac{1}{p_{\rho}^{\prime}} & 0 \\
0 & 0 & 0 & \frac{\kappa_{0}\left(\theta^{\prime}\right)}{\theta^{\prime} p_{\rho}^{\prime}}
\end{array}\right) Q^{-1} \\
& =\left(\begin{array}{cccc}
\frac{1}{p_{\rho}^{\prime}} & 0 & 0 & 0 \\
0 & \frac{\kappa_{0}\left(\theta^{\prime}\right)}{\theta^{\prime} p_{\rho}^{\prime}\left(\alpha^{2}+1\right)} & \frac{-\alpha \kappa_{0}\left(\theta^{\prime}\right)}{\sqrt{2}\left(\alpha^{2}+1\right) \theta^{\prime} p_{\rho}^{\prime}} & \frac{-\alpha \kappa_{0}\left(\theta^{\prime}\right)}{\sqrt{2}\left(\alpha^{2}+1\right) \theta^{\prime} p_{\rho}^{\prime}} \\
0 & \frac{-\alpha \kappa_{0}\left(\theta^{\prime}\right)}{\sqrt{2}\left(\alpha^{2}+1\right) \theta^{\prime} p_{\rho}^{\prime}} & \frac{1}{2 p_{\rho}^{\prime}}+\frac{\alpha^{2} \kappa_{0}\left(\theta^{\prime}\right)}{2\left(\alpha^{2}+1\right) \theta^{\prime} p_{\rho}^{\prime}} & -\frac{1}{2 p_{\rho}^{\prime}}+\frac{\alpha^{2} \kappa_{0}\left(\theta^{\prime}\right)}{2\left(\alpha^{2}+1\right) \theta^{\prime} p_{\rho}^{\prime}} \\
0 & \frac{-\alpha \kappa_{0}\left(\theta^{\prime}\right)}{\sqrt{2}\left(\alpha^{2}+1\right) \theta^{\prime} p_{\rho}^{\prime}} & -\frac{1}{2 p_{\rho}^{\prime}}+\frac{\alpha^{2} \kappa_{0}\left(\theta^{\prime}\right)}{2\left(\alpha^{2}+1\right) \theta^{\prime} p_{\rho}^{\prime}} & \frac{1}{2 p_{\rho}^{\prime}}+\frac{\alpha^{2} \kappa_{0}\left(\theta^{\prime}\right)}{2\left(\alpha^{2}+1\right) \theta^{\prime} p_{\rho}^{\prime}}
\end{array}\right), \\
& \mathcal{G}^{11}=\left(\begin{array}{cccc}
0 & 0 & 0 & 0 \\
0 & 0 & 0 & 0 \\
0 & 0 & \frac{1}{2} & -\frac{1}{2} \\
0 & 0 & -\frac{1}{2} & \frac{1}{2}
\end{array}\right), \quad \mathcal{G}^{22}=\left(\begin{array}{cccc}
1 & 0 & 0 & 0 \\
0 & 0 & 0 & 0 \\
0 & 0 & 0 & 0 \\
0 & 0 & 0 & 0
\end{array}\right) \\
& \mathcal{G}^{12}=\mathcal{G}^{21}=\left(\begin{array}{cccc}
0 & 0 & \frac{\sqrt{2}}{4} & -\frac{\sqrt{2}}{4} \\
0 & 0 & 0 & 0 \\
\frac{\sqrt{2}}{4} & 0 & 0 & 0 \\
-\frac{\sqrt{2}}{4} & 0 & 0 & 0
\end{array}\right)
\end{aligned}
$$

We can also rewrite $\mathcal{L}^{\varepsilon}$ as

$$
\mathcal{L}^{\varepsilon}=\mathcal{L}^{0}+\varepsilon^{2} \Lambda
$$

where $\mathcal{L}^{0}=-\mathcal{A}_{j} \partial_{j}-\mathcal{W}$, and $\Lambda=\left(\mathcal{Q}^{1}+\mathcal{Q}^{2}\right)+\sum_{j=1}^{2}\left(\mathcal{P}_{j}+\mathcal{I}_{j}\right) \partial_{j}+\left(\mathcal{G} \Delta+\sum_{j=1}^{2} \mathcal{G}^{i j} \partial_{i j}\right)$.

The boundary condition of (1.7) can be rewritten in terms of the boundary characteristic variables as:

$$
\mathcal{M}^{+} U=0 \quad \text { on } \Gamma \times[0, T] \quad \text { where } \quad \mathcal{M}^{+}=M^{+} Q^{-1}=\left(\begin{array}{cccc}
0 & 0 & \frac{1}{\sqrt{2}} & \frac{-1}{\sqrt{2}} \\
1 & 0 & 0 & 0 \\
0 & \frac{-1}{\sqrt{\alpha^{2}+1}} & \frac{\alpha}{\sqrt{2\left(\alpha^{2}+1\right)}} & \frac{\alpha}{\sqrt{2\left(\alpha^{2}+1\right)}}
\end{array}\right),
$$

i.e.

$$
\mathrm{u}_{2}-\mathrm{u}_{3}=0, \quad \mathrm{u}_{0}=0, \quad-\mathrm{u}_{1}+\frac{\alpha}{\sqrt{2}} \mathrm{u}_{2}+\frac{\alpha}{\sqrt{2}} \mathrm{u}_{3}=0, \quad \text { on } \quad \Gamma \times[0, T],
$$

which can be simplified as

$$
\mathrm{u}_{2}=\mathrm{u}_{3}, \quad \mathrm{u}_{0}=0, \quad \mathrm{u}_{1}=\sqrt{2} \alpha \mathrm{u}_{2}, \quad \text { on } \quad \Gamma \times[0, T] .
$$

The initial conditions of (1.7) can be rewritten as:

$$
U(x, 0)=Q V_{0}=U_{0}(x), \quad \text { for } \quad x \in \Omega .
$$

Furthermore, the boundary condition for the linearized Euler equations (1.10) becomes

$$
\mathcal{M}^{0} U^{0}=M^{0} Q^{-1} U^{0}=0 \text { on } \Gamma \times[0, T],
$$

i.e.

$$
\mathrm{u}_{2}-\mathrm{u}_{3}=0, \quad \text { on } \Gamma \times[0, T] .
$$

It is clear that the initial data $U_{0}$ satisfies the compatibility condition of order $l \geq 0$ for (2.21), (2.24) and (2.26) for any $\varepsilon>0$ if and only if $V_{0}$ satisfies the compatibility condition of order $l \geq 0$ for any $\varepsilon>0$. The same statement is also true for the linearized Euler case. 
2.2. Formal Inner and Boundary Expansions. We construct the approximate solution $U^{\varepsilon}$ of equation (2.21) with boundary and initial conditions (2.24)-(2.26) as

$$
\begin{aligned}
W^{\varepsilon}(x, t) & =E^{\varepsilon}(x, t)+B^{\varepsilon}(x, t) \\
& =\sum_{i=0}^{N} \varepsilon^{i} E^{i}\left(x_{1}, x_{2}, t\right)+\sum_{i=0}^{N} \varepsilon^{i} B^{i}\left(\frac{x_{1}}{\varepsilon}, x_{2}, t\right) .
\end{aligned}
$$

Formally, for the inner term $E^{\varepsilon}$,

$$
\begin{aligned}
\left(\mathcal{A}_{0} \partial_{t}-\mathcal{L}^{\varepsilon}\right) E^{\varepsilon} & =\left(\mathcal{A}_{0} \partial_{t}-\mathcal{L}^{0}\right) E^{0}+\varepsilon\left(\mathcal{A}_{0} \partial_{t}-\mathcal{L}^{0}\right) E^{1} \\
& +\sum_{i=2}^{N} \varepsilon^{i}\left\{\left(\mathcal{A}_{0} \partial_{t}-\mathcal{L}^{0}\right) E^{i}-\Lambda E^{i-2}\right\}-\varepsilon^{N+1} \Lambda E^{N-1}-\varepsilon^{N+2} \Lambda E^{N} .
\end{aligned}
$$

For the boundary term $B\left(\frac{x_{1}}{\varepsilon}, x_{2}, t\right)$,

$$
\begin{aligned}
\left(\mathcal{A}_{0} \partial_{t}-\mathcal{L}^{\varepsilon}\right) B & =\frac{1}{\varepsilon} \mathcal{A}_{1} \partial_{z_{1}} B+\left\{\mathcal{A}_{0} \partial_{t}+\mathcal{A}_{2} \partial_{z_{2}}-\left(\mathcal{G}+\mathcal{G}^{11}\right) \partial_{z_{1} z_{1}}+\mathcal{W}\right\} B \\
& -\varepsilon\left\{2 \mathcal{G}^{12} \partial_{z_{1} z_{2}}+\left(\mathcal{P}_{1}+\mathcal{I}_{1}\right) \partial_{z_{1}}\right\} B \\
& -\varepsilon^{2}\left\{\left(\mathcal{G}+\mathcal{G}^{22}\right) \partial_{z_{2} z_{2}}+\left(\mathcal{P}_{2}+\mathcal{I}_{2}\right) \partial_{z_{2}}+\left(\mathcal{Q}^{1}+\mathcal{Q}^{2}\right)\right\} B
\end{aligned}
$$

Note that in the above expansion of the differential operator $\mathcal{A}_{0} \partial_{t}-\mathcal{L}^{\varepsilon}$, all the coefficients are functions of $\left(x_{1}, x_{2}, t\right)=\left(\varepsilon z_{1}, z_{2}, t\right)$. In the construction of the boundary layer part of the approximate solution $B^{\varepsilon}$, we only concern the values on the boundary $\Gamma \times[0, T]$. So we expand all the coefficients around $\left(0, z_{2}, t\right)$ as follows: We use the notation that, for a smooth matrix-valued function $\mathcal{K}\left(x_{1}, x_{2}, t\right)=\mathcal{K}\left(\varepsilon z_{1}, z_{2}, t\right)$, the Taylor expansion around $\left(0, z_{2}, t\right)$ is

$$
\begin{aligned}
& \mathcal{K}\left(\varepsilon z_{1}, z_{2}, t\right) \\
= & \left.\sum_{i=0}^{N}\left(\varepsilon z_{1}\right)^{i} \frac{\partial_{x_{1}}^{i} \mathcal{K}\left(0, z_{2}, t\right)}{i !}+\frac{\left(\varepsilon z_{1}\right)^{N+1}}{N !} \int_{0}^{1} \partial_{x_{1}}^{N+1} \mathcal{K}\left(\varepsilon z_{1} \xi, z_{2}, t\right)\right)(1-\xi)^{N} d \xi \\
= & \sum_{i=0}^{N}\left(\varepsilon z_{1}\right)^{i} \mathcal{K}^{i}\left(z_{2}, t\right)+\left(\varepsilon z_{1}\right)^{N+1} \mathcal{K}_{R}^{N+1} .
\end{aligned}
$$

In particular, $\mathcal{A}_{1}\left(\varepsilon z_{1}, z_{2}, t\right)=\mathcal{A}_{1 m}\left(z_{2}, t\right)+\frac{1}{i !} \sum_{i=0}^{N}\left(\varepsilon z_{1}\right)^{i} \partial_{x_{1}}^{i} \mathcal{A}_{1 r}\left(0, z_{2}, t\right)+\left(\varepsilon z_{1}\right)^{N+1}\left(\mathcal{A}_{1 r}^{N+1}\right)_{R}$. Thus

$$
\left(\mathcal{A}_{0} \partial_{t}-\mathcal{L}^{\varepsilon}\right) B=\left\{\frac{1}{\varepsilon} \mathcal{L}_{-1}^{\mathrm{b}}+\mathcal{L}_{0}^{\mathrm{b}}+\varepsilon \mathcal{L}_{1}^{\mathrm{b}}+\varepsilon^{2} \mathcal{L}_{2}^{\mathrm{b}}\right\} B+\text { higher order terms },
$$

where

$$
\begin{aligned}
& \mathcal{L}_{-1}^{\mathrm{b}}=\mathcal{A}_{1}\left(z_{2}, t\right) \partial_{z_{1}}, \\
& \mathcal{L}_{0}^{\mathrm{b}}=\mathcal{A}_{0}\left(z_{2}, t\right) \partial_{t}+\mathcal{A}_{2}\left(z_{2}, t\right) \partial_{z_{2}}-\left(\mathcal{G}+\mathcal{G}^{11}\right)\left(z_{2}, t\right) \partial_{z_{1} z_{1}}^{2}+\mathcal{W}\left(z_{2}, t\right)+z_{1} \partial_{x_{1}} \mathcal{A}_{1 r}\left(z_{2}, t\right) \partial_{z_{1}}, \\
& \mathcal{L}_{1}^{\mathrm{b}}=2 \mathcal{G}^{12}\left(z_{2}, t\right) \partial_{z_{1} z_{2}}^{2}+\left(\mathcal{P}_{1}+\mathcal{I}_{1}+\frac{1}{2} z_{1}^{2} \partial_{x_{1}}^{2} \mathcal{A}_{1 r}\right)\left(z_{2}, t\right) \partial_{z_{1}}+z_{1} \partial_{x_{1}} \mathcal{A}_{0}\left(z_{2}, t\right) \partial_{t}, \\
& \mathcal{L}_{2}^{\mathrm{b}}=\left(\mathcal{G}+\mathcal{G}^{22}\right)\left(z_{2}, t\right) \partial_{z_{2} z_{2}}^{2}+\left(\mathcal{P}_{2}+\mathcal{I}_{2}\right)\left(z_{2}, t\right) \partial_{z_{2}}+\frac{1}{6} z_{1}^{3} \partial_{x_{1}}^{3} \mathcal{A}_{1 r}\left(z_{2}, t\right) \partial_{z_{1}}+\frac{1}{2} z_{1}^{2} \partial_{x_{1}}^{2} \mathcal{A}_{1 r}\left(z_{2}, t\right) \partial_{t} .
\end{aligned}
$$

Here we use the notation $\mathcal{K}\left(z_{2}, t\right)=\mathcal{K}\left(0, z_{2}, t\right)$ for a function $\mathcal{K}$. In (2.32), the precise forms of the "higher order term" are lengthy and not important for the later analysis, so we omit writing out the details. In fact, only $\mathcal{L}_{-1}^{\mathrm{b}}$ and $\mathcal{L}_{0}^{\mathrm{b}}$ play important roles in the later boundary layer analysis. Note that

$$
\mathcal{A}_{2}\left(z_{2}, t\right)=\sqrt{\frac{\alpha^{2}+1}{2}}\left(\begin{array}{cccc}
0 & 0 & 1 & 1 \\
0 & 0 & 0 & 0 \\
1 & 0 & 0 & 0 \\
1 & 0 & 0 & 0
\end{array}\right)
$$


Thus,

$$
\begin{aligned}
& \left(\mathcal{A}_{0} \partial_{t}-\mathcal{L}^{\varepsilon}\right) B^{\varepsilon} \\
= & \frac{1}{\varepsilon} \mathcal{L}_{-1}^{\mathrm{b}} B^{0}+\left\{\mathcal{L}_{-1}^{\mathrm{b}} B^{1}+\mathcal{L}_{0}^{\mathrm{b}} B^{0}\right\}+\varepsilon\left\{\mathcal{L}_{-1}^{\mathrm{b}} B^{2}+\mathcal{L}_{0}^{\mathrm{b}} B^{1}+\mathcal{L}_{1}^{\mathrm{b}} B^{0}\right\} \\
+ & \sum_{i=2}^{N-1} \varepsilon^{i}\left\{\mathcal{L}_{-1}^{\mathrm{b}} B^{i+1}+\mathcal{L}_{0}^{\mathrm{b}} B^{i}+\mathcal{L}_{1}^{\mathrm{b}} B^{i-1}+\mathcal{L}_{2}^{\mathrm{b}} B^{i-2}\right\} \\
+ & \varepsilon^{N}\left\{\mathcal{L}_{0}^{\mathrm{b}} B^{N}+\mathcal{L}_{1}^{\mathrm{b}} B^{N-1}+\mathcal{L}_{2}^{\mathrm{b}} B^{N-2}\right\}+\varepsilon^{N+1}\left\{\mathcal{L}_{1}^{\mathrm{b}} B^{N}+\mathcal{L}_{2}^{\mathrm{b}} B^{N-1}\right\}+\varepsilon^{N+2} \mathcal{L}_{2}^{\mathrm{b}} B^{N} \\
+ & \text { higher order terms . }
\end{aligned}
$$

2.3. Construction of Inner and Boundary Expansions. We now construct the inner and boundary expansions at each order in details. We plug $W^{\varepsilon}=E^{\varepsilon}+B^{\varepsilon}$ into the equation (2.21) and compare the coefficients of the same order in both inner and boundary terms. It should be noted that the same order of inner and boundary functions will be constructed simultaneously due to their coupling at the boundary.

\section{Order $O\left(\frac{1}{\varepsilon}\right)$ of boundary expansion:}

We start from the first-order term $B^{0}$ in the boundary expansion by setting the order $O\left(\frac{1}{\varepsilon}\right)$ in the boundary part zero gives $\mathcal{L}_{-1}^{\mathrm{b}} B^{0}=0$, i.e

$$
\mathcal{A}_{1}\left(0, z_{2}, t\right) \partial_{z_{1}} B^{0}\left(z_{1}, z_{2}, t\right)=0 .
$$

Note that $\mathcal{A}_{1}\left(0, z_{2}, t\right)=\mathcal{A}_{1 m}\left(0, z_{2}, t\right)$ since $\mathcal{A}_{1 r}$ vanishes on the boundary $\Gamma \times[0, T]$, i.e. $\mathcal{A}_{1 r}\left(0, z_{2}, t\right)=$ 0 . Noting (2.23), the equation (2.35) is equivalent to

$$
\left(\begin{array}{cc}
\sqrt{\alpha^{2}+1} & 0 \\
0 & -\sqrt{\alpha^{2}+1}
\end{array}\right)\left(\begin{array}{c}
\partial_{z_{1}} B_{2}^{0} \\
\partial_{z_{1}} B_{3}^{0}
\end{array}\right)=\left(\begin{array}{l}
0 \\
0
\end{array}\right) \quad \text { in } \quad \Omega \times[0, T] .
$$

Note that we impose the decay condition at infinity that

$$
B_{j}^{0}\left(z_{1}, z_{2}, t\right) \rightarrow 0 \quad \text { as } \quad z_{1} \rightarrow \infty, \quad\left(z_{2}, t\right) \in \mathbb{R} \times[0, T] .
$$

The only solution of (2.36) and (2.37) is given as

$$
B_{2}^{0}\left(z_{1}, z_{2}, t\right)=B_{3}^{0}\left(z_{1}, z_{2}, t\right) \equiv 0 \quad \text { for } \quad z_{1} \geq 0, \quad\left(z_{2}, t\right) \in \mathbb{R} \times[0, T] .
$$

\section{Order $O(1)$ of inner expansion:}

We determine the leading order term $E^{0}$ in the inner expansion by setting the $O(1)$-order term in (2.30) to zero and then equipping the resulting equations with the same initial and boundary conditions as in (2.24) and (2.27). So we deduce the following initial boundary value problem for $E^{0}$ :

$$
\begin{aligned}
& \mathcal{A}_{0} \partial_{t} E^{0}-\mathcal{L} E^{0}=0, \quad \text { in } \Omega \times[0, T], \\
& \mathcal{M}^{0}\left(E^{0}+B^{0}\right)=0, \quad \text { on } \Gamma \times[0, T] \text {, } \\
& E^{0}(x, 0)=U_{0}(x), \text { for } x \in \Omega .
\end{aligned}
$$

Note that from the definition of $\mathcal{M}^{0}$, see (2.28), only the third and fourth components of $B^{0}$ and $E^{0}$ are involved, and $B_{2}^{0}$ and $B_{3}^{0}$ are solved in (2.38). Thus the boundary condition for $E^{0}$ in (2.39) is $\mathcal{M}^{0} E^{0}=0$, more specifically, $E_{2}^{0}-E_{3}^{0}=0$.

It is easy to see that $Q^{-1} E^{0}$ is a solution of the initial boundary value problem of the linearized Euler equations with the same boundary and initial conditions as in (1.10). Then it follows by Proposition 4.2 that there exists a unique $E^{0}$ of the problem (2.39), such that

$$
E^{0} \in \bigcap_{j=0}^{m} C^{j}\left([0, T] ; H^{m-j}(\Omega)\right) .
$$

\section{Order $O(1)$ of boundary expansion:}


By setting the term of $O(1)$ in (2.34) equal to zero, we have

$$
\mathcal{L}_{0}^{\mathrm{b}} B^{0}+\mathcal{L}_{-1}^{\mathrm{b}} B^{1}=0,
$$

which gives

$$
\begin{aligned}
& \left(\begin{array}{cccc}
\frac{\rho^{\prime}}{p_{\rho}^{\prime}} & 0 & 0 & 0 \\
0 & \eta_{0} & \eta_{1} & \eta_{1} \\
0 & \eta_{1} & \eta_{2} & \eta_{3} \\
0 & \eta_{1} & \eta_{3} & \eta_{2}
\end{array}\right) \partial_{t}\left(\begin{array}{c}
B_{0}^{0} \\
B_{1}^{0} \\
0 \\
0
\end{array}\right)+\sqrt{\frac{\alpha^{2}+1}{2}}\left(\begin{array}{cccc}
0 & 0 & 1 & 1 \\
0 & 0 & 0 & 0 \\
1 & 0 & 0 & 0 \\
1 & 0 & 0 & 0
\end{array}\right) \partial_{z_{2}}\left(\begin{array}{c}
B_{0}^{0} \\
B_{1}^{0} \\
0 \\
0
\end{array}\right) \\
- & \left(\begin{array}{cccc}
\frac{1}{p_{\rho}^{\prime}} & 0 & 0 & 0 \\
0 & \tau_{0} & \tau_{1} & \tau_{1} \\
0 & \tau_{1} & \tau_{2} & \tau_{3} \\
0 & \tau_{1} & \tau_{3} & \tau_{2}
\end{array}\right) \partial_{z_{1} z_{1}}^{2}\left(\begin{array}{c}
B_{0}^{0} \\
B_{1}^{0} \\
0 \\
0
\end{array}\right)+\left\{\mathcal{W}\left(z_{2}, t\right)+z_{1} \partial_{x_{1}} \mathcal{A}_{1 r}\left(z_{2}, t\right) \partial_{z_{1}}\right\}\left(\begin{array}{c}
B_{0}^{0} \\
B_{1}^{0} \\
0 \\
0
\end{array}\right) \\
= & -\left(\begin{array}{cccc}
0 & 0 & 0 & 0 \\
0 & 0 & 0 & 0 \\
0 & 0 & \sqrt{\alpha^{2}+1} & 0 \\
0 & 0 & 0 & -\sqrt{\alpha^{2}+1}
\end{array}\right) \partial_{z_{1}}\left(\begin{array}{c}
B_{0}^{1} \\
B_{1}^{1} \\
B_{2}^{1} \\
B_{3}^{1}
\end{array}\right) .
\end{aligned}
$$

Noticing that the second term in the first line of (2.41) vanishes, i.e. there are no $\partial_{z_{2}}$ terms in (2.41), the first two components of (2.41) give the equations of $B_{0}^{0}$ and $B_{1}^{0}$ which are Prandtl-type linearly coupled equations:

$$
\begin{aligned}
\frac{\rho^{\prime}}{p_{\rho}^{\prime}}\left(z_{2}, t\right) \partial_{t} B_{0}^{0}-\frac{1}{p_{\rho}^{\prime}}\left(z_{2}, t\right) \partial_{z_{1} z_{1}}^{2} B_{0}^{0} & +\left(a_{11}^{r} z_{1} \partial_{z_{1}} B_{0}^{0}+a_{12}^{r} z_{1} \partial_{z_{1}} B_{1}^{0}\right) \\
+ & \left(w_{11} B_{0}^{0}+w_{12} B_{1}^{0}\right)=0
\end{aligned}
$$

and

$$
\begin{aligned}
\eta_{0}\left(z_{2}, t\right) \partial_{t} B_{1}^{0}-\tau_{0}\left(z_{2}, t\right) \partial_{z_{1} z_{1}}^{2} B_{1}^{0} & +\left(a_{21}^{r} z_{1} \partial_{z_{1}} B_{0}^{0}+a_{22}^{r} z_{1} \partial_{z_{1}} B_{1}^{0}\right) \\
& +\left(w_{21} B_{0}^{0}+w_{22} B_{1}^{0}\right)=0
\end{aligned}
$$

with the boundary conditions

$$
B_{0}^{0}=-E_{0}^{0}, \quad B_{1}^{0}=-E_{1}^{0}+\sqrt{2} \alpha E_{2}^{0} \quad \text { on } \quad \Gamma \times[0, T],
$$

and the initial conditions

$$
B_{0}^{0}(z, 0)=B_{1}^{0}(z, 0)=0 \quad \text { for } \quad z \in \Omega .
$$

We denote (2.42) and (2.43) as

$$
\mathcal{E}\left(B_{0}^{0}, B_{1}^{0}\right)=0 .
$$

To solve (2.46) we need to verify the compatibility condition. So we set

$$
\begin{aligned}
\tilde{B}_{0}^{0} & =B_{0}^{0}\left(z_{1}, z_{2}, t\right)+E_{0}^{0}\left(0, z_{2}, t\right) e^{-z_{1}^{2}}=B_{0}^{0}+\tilde{E}_{0}^{0}, \\
\tilde{B}_{1}^{0} & =B_{1}^{0}\left(z_{1}, z_{2}, t\right)+\left(E_{1}^{0}\left(0, z_{2}, t\right)-\sqrt{2} \alpha E_{2}^{0}\left(0, z_{2}, t\right)\right) e^{-z_{1}^{2}} \\
& =B_{1}^{0}+\tilde{E}_{1}^{0} .
\end{aligned}
$$

Then $\left(\tilde{B}_{0}^{0}, \tilde{B}_{1}^{0}\right)$ satisfies

$$
\mathcal{E}\left(\tilde{B}_{0}^{0}, \tilde{B}_{1}^{0}\right)=\mathcal{E}\left(\tilde{E}_{0}^{0}, \tilde{E}_{1}^{0}\right) .
$$

As $V_{0}$ satisfies the compatibility condition of order $\left[\frac{m}{2}\right]-1$, for the problem (1.7) for any $\varepsilon>0$, one derive that

$$
\partial_{t}^{k} \tilde{E}_{i}^{0}(z, 0)=0, \quad k=0,1, \cdots,\left[\frac{m}{2}\right]-1,
$$

and one also could check that

$$
\left\langle z_{1}\right\rangle^{l} \partial_{t}^{k} \partial_{z_{1}}^{\alpha_{1}} \partial_{z_{2}}^{\alpha_{2}}\left[\mathcal{E}\left(\tilde{E}_{0}^{0}, \tilde{E}\right)\right] \in C^{0}\left([0, T] ; L^{2}(\Omega)\right) \text { for } \quad k+|\alpha| \leq m-3,
$$


Then by Proposition 4.3 which will be presented in the last section, we obtain a unique solution $\left(B_{1}^{0}, B_{1}^{0}\right)$ to (2.42)-(2.43)-(2.44)-(2.45) such that

$$
\left\langle z_{1}\right\rangle^{l} \partial_{t}^{k} \partial_{z_{1}}^{\alpha_{1}} \partial_{z_{2}}^{\alpha_{2}} \tilde{B}_{i}^{0} \in C^{0}\left([0, T] ; L^{2}(\Omega)\right), \quad i=0,1,
$$

for $k+|\alpha| \leq m-4, k+\left[\frac{\alpha_{1}+1}{2}\right] \leq\left[\frac{m}{2}\right]-2$, and

$$
\partial_{t}^{k} \tilde{B}_{i}^{0}(z, 0)=0, \quad \text { for } \quad k=0,1, \cdots,\left[\frac{m}{2}\right]-2, \quad i=0,1 .
$$

Thus we have

$$
\left\langle z_{1}\right\rangle^{l} \partial_{t}^{k} \partial_{z_{1}}^{\alpha_{1}} \partial_{z_{2}}^{\alpha_{2}} B_{i}^{0} \in C^{0}\left([0, T] ; L^{2}(\Omega)\right) \quad i=0,1,
$$

for $k+|\alpha| \leq m-4, k+\left[\frac{\alpha_{1}+1}{2}\right] \leq\left[\frac{m}{2}\right]-2$, and

$$
\partial_{t}^{k} B_{i}^{0}(z, 0)=0, \quad \text { for } \quad k=0,1, \cdots,\left[\frac{m}{2}\right]-2, \quad i=0,1 .
$$

The third and the fourth equations of (2.41) could be written as the following ODEs for $z_{1}$ $\left(z_{2}\right.$ and $t$ are parameters):

$$
\left(\begin{array}{cc}
\sqrt{\alpha^{2}+1} & 0 \\
0 & -\sqrt{\alpha^{2}+1}
\end{array}\right) \partial_{z_{1}}\left(\begin{array}{l}
B_{2}^{1} \\
B_{3}^{1}
\end{array}\right)\left(z_{1}, z_{2}, t\right)=\left(\begin{array}{l}
H_{2}^{1}\left(B^{0}\right) \\
H_{3}^{1}\left(B^{0}\right)
\end{array}\right)\left(z_{1}, z_{2}, t\right),
$$

where $H_{j}^{1}(j=2,3)$ are linear functions of the known functions $B_{0}^{0}$ and $B_{1}^{0}$. By the condition (2.37), we have

$$
B_{j}^{1}\left(z_{1}, z_{2}, t\right)=\int_{z_{1}}^{\infty}(-1)^{j} \sqrt{\alpha^{2}+1} H_{j}^{1}\left(B^{0}\right)\left(\xi, z_{2}, t\right) \mathrm{d} \xi, \quad j=2,3 .
$$

It follows from (2.47) that

$$
\left\langle z_{1}\right\rangle^{l} \partial_{t}^{k} \partial_{z_{1}}^{\alpha_{1}} \partial_{z_{2}}^{\alpha_{2}} B_{i}^{1} \in C^{0}\left([0, T] ; L^{2}(\Omega)\right) \quad i=2,3,
$$

for $k+|\alpha| \leq m-5, k+\left[\frac{\alpha_{1}+1}{2}\right] \leq\left[\frac{m}{2}\right]-2$, and

$$
\partial_{t}^{k} B_{i}^{1}(z, 0)=0, \quad \text { for } \quad k=0,1, \cdots,\left[\frac{m}{2}\right]-3, \quad i=2,3 .
$$

\section{Order $O(\varepsilon)$ of inner expansion:}

By setting the order $O(\varepsilon)$ in the inner expansion (2.30), we are led to the follow initial boundary value problem of $E^{1}$ :

$$
\begin{aligned}
& \left(\mathcal{A}_{0} \partial_{t}-\mathcal{L}^{0}\right) E^{1}=0, \quad \text { in } \Omega \times[0, T], \\
& \mathcal{M}^{0}\left(E^{1}+B^{1}\right)=0, \quad \text { on } \Gamma \times[0, T] \\
& E^{1}(z, 0)=0, \quad \text { for } z \in \Omega .
\end{aligned}
$$

Again, note that $\mathcal{M}^{0} B^{1}$ does not contain $B_{0}^{1}$ and $B_{1}^{1}$, only contains $B_{2}^{1}$ and $B_{3}^{1}$ which are solved in the last step, see (2.50). So the boundary condition $\mathcal{M}^{0} E^{1}=-\mathcal{M}^{0} B^{1}$ is known, i.e. $E_{2}^{1}-E_{3}^{1}=-B_{2}^{1}+B_{3}^{1}$ on $\Gamma \times[0, T]$. To obtain the existence of the unique solution $E^{1}$ to (2.53), we need verify the compatibility conditions. We set

$$
\tilde{E}^{1}=E^{1}-\left(0,0, B_{2}^{1}\left(0, z_{2}, t\right), B_{3}^{1}\left(0, z_{2}, t\right)\right)^{\top} e^{-z_{1}^{2}}=E^{1}-\bar{B}^{1} .
$$

It follows from (2.53) and (2.52), $\tilde{E}^{1}$ satisfies the equation

$$
\begin{aligned}
\left(\mathcal{A}_{0} \partial_{t}-\mathcal{L}^{0}\right) \tilde{E}^{1} & =-\left(\mathcal{A}_{0} \partial_{t}-\mathcal{L}^{0}\right) \bar{B}^{1}, \quad \text { in } \quad \Omega \times[0, T], \\
\tilde{E}_{2}^{1}-\tilde{E}_{3}^{1} & =0, \quad \text { on } \Gamma \times[0, T] \\
\tilde{E}^{1}(x, 0) & =0, \quad \text { for } \quad x \in \Omega .
\end{aligned}
$$

It follows from (2.51) that

$$
\left(\mathcal{A}_{0} \partial_{t}-\mathcal{L}^{0}\right) \bar{B}^{1} \in H^{\left[\frac{m}{2}\right]-4}(\Omega \times[0, T])
$$


and $\partial_{t}^{k} \mathcal{F}\left(\bar{B}^{1}\right)(x, 0)=0, k=0,1, \cdots,\left[\frac{m}{2}\right]-4$, then by Proposition 4.2, there exists a unique solution to (2.54)

$$
\tilde{E}^{1} \in \bigcap_{j=0}^{\left[\frac{m}{2}\right]-4} C^{j}\left([0, T] ; H^{\left[\frac{m}{2}\right]-4-j}\right),
$$

this gives a unique solution $E^{1}$ to (2.53) such that

$$
E^{1} \in \bigcap_{j=0}^{\left[\frac{m}{2}\right]-4} C^{j}\left([0, T] ; H^{\left[\frac{m}{2}\right]-4-j}\right) .
$$

\section{Order $O(\varepsilon)$ of boundary expansion:}

Similar as before, we next construct $\left(B_{0}^{1}, B_{1}^{1}\right)$ and $\left(B_{2}^{2}, B_{3}^{2}\right)$ by setting the $O(\varepsilon)$-order term in (2.34) equal to zero which gives

$$
\begin{aligned}
\mathcal{L}_{-1}^{\mathrm{b}} B^{2}+\mathcal{L}_{0}^{\mathrm{b}} B^{1} & =-\mathcal{L}_{1}^{\mathrm{b}} B^{0}, \quad \text { in } \quad \Omega \times[0, T] \\
\mathcal{M}^{+}\left(B^{1}+E^{1}\right) & =0, \quad \text { on } \Gamma \times[0, T] \\
B^{1}(z, 0) & =0, \quad \text { for } \quad z \in \Omega .
\end{aligned}
$$

The first two components of (2.55) are a linear system of Prandtl-type equations

$$
\mathcal{E}\left(B_{0}^{1}, B_{1}^{1}\right)=F_{1}\left(B^{0},\left(B^{1}\right)_{\mathrm{II}}\right),
$$

where the inhomogeneous term $F_{1}\left(B^{0},\left(B^{1}\right)_{\text {II }}\right)$ is a two components vector-valued function:

$$
\begin{aligned}
F_{1}\left(B^{0},\left(B^{1}\right)_{\mathrm{II}}\right)= & -\left(\begin{array}{c}
0 \\
\eta_{1} \partial_{t}\left(B_{2}^{1}+B_{3}^{1}\right)
\end{array}\right)-\sqrt{\frac{\alpha^{2}+1}{2}}\left(\begin{array}{c}
\partial_{z_{2}}\left(B_{2}^{1}+B_{3}^{1}\right) \\
0
\end{array}\right)+\left(\begin{array}{c}
0 \\
\tau_{1} \partial_{z_{1} z_{1}}^{2}\left(B_{2}^{1}+B_{3}^{1}\right)
\end{array}\right) \\
& +\left(\mathcal{W}+z_{1} \partial_{x_{1}} \mathcal{A}_{1 r}\right) \partial_{z_{1}}\left(0,0, B_{2}^{1}, B_{3}^{1}\right)^{\top}-\left(\mathcal{L}_{1}^{\mathrm{b}} B^{0}\right)_{\mathrm{I}} .
\end{aligned}
$$

Here we use the notation that for a four components vector $U, U_{\text {I }}$ denotes the first two components, and $U_{\text {II }}$ denotes the last two components. Note that $E^{1}$ is already solved in the last step, so the boundary and initial condition of (2.56) are

$$
\begin{array}{r}
B_{0}^{1}=-E_{0}^{1}, \quad B_{1}^{1}=-E_{1}^{1}+\sqrt{2} \alpha E_{2}^{1}, \quad \text { on } \Gamma \times[0, T], \\
\left(B_{0}^{1}, B_{1}^{1}\right)^{\top}(z, 0)=(0,0)^{\top}, \quad z \in \Omega .
\end{array}
$$

Similar as in solving $\left(B_{0}^{0}, B_{0}^{1}\right)$, set

$$
\begin{aligned}
\tilde{B}_{0}^{1} & =B_{0}^{1}\left(z_{1}, z_{2}, t\right)+E_{0}^{1}\left(0, z_{2}, t\right) e^{-z_{1}^{2}}=B_{0}^{1}+\tilde{E}_{0}^{1}, \\
\tilde{B}_{1}^{1} & =B_{1}^{1}\left(z_{1}, z_{2}, t\right)+\left(E_{1}^{1}\left(0, z_{2}, t\right)-\sqrt{2} \alpha E_{2}^{1}\left(0, z_{2}, t\right)\right) e^{-z_{1}^{2}} \\
& =B_{1}^{1}+\tilde{E}_{1}^{1},
\end{aligned}
$$

Then Proposition 4.3 shows that there exists a unique solution $\left(B_{0}^{1}, B_{1}^{1}\right)$ to (2.56)-(2.58) such that

$$
\left\langle z_{1}\right\rangle^{l} \partial_{t}^{k} \partial_{z_{1}}^{\alpha_{1}} \partial_{z_{2}}^{\alpha_{2}} B_{i}^{1} \in C^{0}\left([0, T] ; L^{2}(\Omega)\right) \quad i=0,1, \quad k+|\alpha| \leq\left[\frac{m}{2}\right]-8
$$

and

$$
\partial_{t}^{k} B_{i}^{1}(z, 0)=0 \quad i=0,1, \quad k=0,1, \cdots,\left[\frac{m}{2}\right]-8 .
$$

The third and fourth components of (2.55) are ODEs for $\left(B_{2}^{2}, B_{3}^{2}\right)$ :

$$
\left(\begin{array}{cc}
\sqrt{\alpha^{2}+1} & 0 \\
0 & -\sqrt{\alpha^{2}+1}
\end{array}\right) \partial_{z_{1}}\left(\begin{array}{l}
B_{2}^{2} \\
B_{3}^{2}
\end{array}\right)\left(z_{1}, z_{2}, t\right)=\left(\begin{array}{l}
H_{2}^{2}\left(B^{0}, B^{1}\right) \\
H_{3}^{2}\left(B^{0}, B^{1}\right)
\end{array}\right)\left(z_{1}, z_{2}, t\right)
$$


where $H_{j}^{2}(j=2,3)$ are linear functions of the known functions $B^{0}$ and $B^{1}$. The equation (2.60) is regarded as an ordinary equation for $B_{j}^{2}$ for $j=2,3$ with independent variable $z_{1}$ and parameters $\left(z_{2}, t\right) \in \mathbb{R} \times[0, T]$. The condition at infinity are imposed as

$$
B_{j}^{2}\left(z_{1}, z_{2}, t\right) \rightarrow 0 \quad \text { as } \quad z_{1} \rightarrow \infty, \quad\left(z_{2}, t\right) \in \mathbb{R} \times[0, T] .
$$

Thus, the solution to (2.60)-(2.61) are uniquely given by

$$
B_{j}^{2}\left(z_{1}, z_{2}, t\right)=\int_{z_{1}}^{\infty}(-1)^{j} \sqrt{\alpha^{2}+1} H_{j}^{2}\left(B^{0}, B^{1}\right)\left(\xi, z_{2}, t\right) \mathrm{d} \xi, \quad j=2,3
$$

and they have the following properties

$$
\left\langle z_{1}\right\rangle^{l} \partial_{t}^{k} \partial_{z_{1}}^{\alpha_{1}} \partial_{z_{2}}^{\alpha_{2}} B_{j}^{2} \in C^{0}\left([0, T] ; L^{2}(\Omega)\right) \quad j=2,3, \quad k+|\alpha| \leq\left[\frac{m}{2}\right]-9 .
$$

\section{General cases:}

For general $i \geq 2$, to solve $E^{i}$ and $B^{i}$, it includes 3 steps: The first step is to solve $\left(B_{2}^{i}, B_{3}^{i}\right)$ by ODEs which comes from the third and fourth components of equation $\mathcal{L}_{-1}^{\mathrm{b}} B^{i}+\mathcal{L}_{0}^{\mathrm{b}} B^{i-1}+$ $\mathcal{L}_{1}^{\mathrm{b}} B^{i-2}+\mathcal{L}_{2}^{\mathrm{b}} B^{i-3}=0$ :

$$
\left(\begin{array}{cc}
\sqrt{\alpha^{2}+1} & 0 \\
0 & -\sqrt{\alpha^{2}+1}
\end{array}\right) \partial_{z_{1}}\left(\begin{array}{c}
B_{2}^{i} \\
B_{3}^{i}
\end{array}\right)\left(z_{1}, z_{2}, t\right)=\left(\begin{array}{l}
H_{2}^{i}\left(B^{0}, B^{1}, \cdots, B^{i-1}\right) \\
H_{3}^{i}\left(B^{0}, B^{1}, \cdots, B^{i-1}\right)
\end{array}\right)\left(z_{1}, z_{2}, t\right)
$$

with the condition at infinity

$$
B_{j}^{i}\left(z_{1}, z_{2}, t\right) \rightarrow 0 \quad \text { as } \quad z_{1} \rightarrow \infty, \quad\left(z_{2}, t\right) \in \mathbb{R} \times[0, T] .
$$

Thus

$$
B_{j}^{i}=\int_{z_{1}}^{\infty}(-1)^{j} \sqrt{\alpha^{2}+1} H_{j}^{2}\left(B^{0}, B^{1}, \cdots, B^{i-1}\right)\left(\xi, z_{2}, t\right) d \xi, \quad j=2,3
$$

and they have the following properties

$$
\left\langle z_{1}\right\rangle^{l} \partial_{t}^{k} \partial_{z_{1}}^{\alpha_{1}} \partial_{z_{2}}^{\alpha_{2}} B_{j}^{i} \in C^{0}\left([0, T] ; L^{2}(\Omega)\right) \quad j=2,3, \quad k+|\alpha| \leq\left[\frac{m}{2}\right]+5-7 i .
$$

The second step is to solve $E^{i}$ by setting the order $O\left(\varepsilon^{i}\right)$ in the inner expansion (2.30) to zero to derive the equation of $E^{i}$ which is a linearized Euler equation with inhomogeneous term:

$$
\begin{aligned}
\left(\mathcal{A}_{0} \partial_{t}-\mathcal{L}^{0}\right) E^{i}=\Lambda E^{i-2}, & \text { in } \Omega \times[0, T], \\
\mathcal{M}^{0} E^{i}=\mathcal{M}^{0} B^{i}, & \text { on } \Gamma \times[0, T], \\
E^{1}(z, 0)=0, & \text { for } z \in \Omega .
\end{aligned}
$$

Since $\mathcal{M}^{0} B^{i}$ does not contain $B_{0}^{i}$ and $B_{1}^{i}$, only contains $B_{2}^{i}$ and $B_{3}^{i}$ which are solved in the last step, see (2.66). So the boundary condition $\mathcal{M}^{0} E^{i}=-\mathcal{M}^{0} B^{i}$ is known, i.e. $E_{2}^{i}-E_{3}^{i}=-B_{2}^{i}+B_{3}^{i}$ on $\Gamma \times[0, T]$. Similar as before, after verifying the compatibility conditions, we obtain a unique solution $E^{i}$ to the initial boundary problem (2.68) by Proposition 4.2.

$$
E^{i} \in \bigcap_{j=0}^{\left[\frac{m}{2}\right]+3-7 i} C^{j}\left([0, T] ; H^{\left[\frac{m}{2}\right]+3-7 i-j}(\Omega)\right) .
$$

The third step is to solve $\left(B_{0}^{i}, B_{1}^{i}\right)$ by setting the $O\left(\varepsilon^{i}\right)$-order term in (2.34) equal to zero which gives

$$
\begin{aligned}
\mathcal{L}_{-1}^{\mathrm{b}} B^{i+1}+\mathcal{L}_{0}^{\mathrm{b}} B^{i} & =-\left(\mathcal{L}_{1}^{\mathrm{b}} B^{i-1}+\mathcal{L}_{1}^{\mathrm{b}} B^{i-2}\right), \\
\mathcal{M}^{+}\left(B^{i}+E^{i}\right) & =0, \quad \text { on } \Gamma \times[0, T] \\
B^{i}(z, 0) & =0, \quad \text { for } \quad z \in \Omega \times[0, T]
\end{aligned}
$$


The first two components of (2.69) are a linear system of Prandtl-type equations

$$
\mathcal{E}\left(B_{0}^{i}, B_{1}^{i}\right)=F_{i}\left(B^{0}, B^{1}, \cdots, B^{i-1},\left(B^{i}\right)_{\mathrm{II}}\right)
$$

where the inhomogeneous term $F_{i}\left(B^{0}, B^{1}, \cdots, B^{i-1},\left(B^{i}\right)\right.$ II $)$ is a two components vector-valued function:

$$
\begin{aligned}
& F_{i}\left(B^{0}, B^{1}, \cdots, B^{i-1},\left(B^{i}\right)_{\mathrm{II}}\right) \\
= & -\left(\begin{array}{c}
0 \\
\eta_{1} \partial_{t}\left(B_{2}^{i}+B_{3}^{i}\right)
\end{array}\right)-\sqrt{\frac{\alpha^{2}+1}{2}}\left(\begin{array}{c}
\partial_{z_{2}}\left(B_{2}^{i}+B_{3}^{i}\right) \\
0
\end{array}\right)+\left(\begin{array}{c}
0 \\
\tau_{1} \partial_{z_{1} z_{1}}^{2}\left(B_{2}^{i}+B_{3}^{i}\right)
\end{array}\right) \\
& +\left(\mathcal{W}+z_{1} \partial_{x_{1}} \mathcal{A}_{1 r}\right) \partial_{z_{1}}\left(0,0, B_{2}^{i}, B_{3}^{i}\right)^{\top}-\left(\mathcal{L}_{1}^{\mathrm{b}} B^{i-1}+\mathcal{L}_{2}^{\mathrm{b}} B^{i-2}\right)_{\mathrm{I}} .
\end{aligned}
$$

Note that $E^{1}$ is already solved in the last step, so the boundary and initial condition of (2.56) are

$$
\begin{array}{r}
B_{0}^{i}=-E_{0}^{1}, \quad B_{1}^{i}=-E_{1}^{i}+\sqrt{2} \alpha E_{2}^{i}, \quad \text { on } \Gamma \times[0, T], \\
\left(B_{0}^{i}, B_{1}^{i}\right)^{\top}(z, 0)=(0,0)^{\top}, \quad z \in \Omega .
\end{array}
$$

Then Proposition 4.3 shows that there exists a unique solution $\left(B_{0}^{i}, B_{1}^{i}\right)$ to (2.70)-(2.72) such that

$$
\left\langle z_{1}\right\rangle^{l} \partial_{t}^{k} \partial_{z_{1}}^{\alpha_{1}} \partial_{z_{2}}^{\alpha_{2}} B_{j}^{i} \in C^{0}\left([0, T] ; L^{2}(\Omega)\right) \text { for } k+|\alpha| \leq\left[\frac{m}{2}\right]-1-7 i
$$

The same as before, the third and fourth components of (2.69) are ODEs for $\left(B_{2}^{i+1}, B_{3}^{i+1}\right)$. Then we can continue the process and solve all $B^{j}$ and $E^{j}$ for $j=0,1, \cdots, N$ for any $N \in \mathbb{N}$.

2.4. Error Terms. We can conclude that the approximate solution $W^{\varepsilon}(x, t)$ for $\varepsilon>0$ in (2.29) has at least the smoothness such that

$$
W^{\varepsilon}(x, t) \in \bigcap_{j=0}^{[m / 2]-1-7 N} C^{j}\left([0, T] ; H^{[m / 2]-1-7 N-j}(\Omega)\right) .
$$

and $W^{\varepsilon}$ satisfies the equation

$$
\left(\mathcal{A}_{0}-\mathcal{L}^{\varepsilon}\right) W^{\varepsilon}\left(x_{1}, x_{2}, t\right)=\varepsilon^{N} g_{B}^{\varepsilon}\left(\frac{x_{1}}{\varepsilon}, x_{2}, t\right)+\varepsilon^{N+1} g_{E}^{\varepsilon}(x, t),
$$

for $(x, t) \in \Omega \times[0, T]$, with the boundary and initial conditions

$$
\begin{array}{r}
W_{2}^{\varepsilon}-W_{3}^{\varepsilon}=0, \quad W_{0}^{\varepsilon}=0, \quad-W_{1}^{\varepsilon}+\sqrt{2} \alpha W_{2}^{\varepsilon}=0, \\
W^{\varepsilon}(x, 0)=U_{0}(x), \quad \text { on } \quad \text { for } \quad x \in[0, T),
\end{array}
$$

where the precise expressions of $g_{E}^{\varepsilon}(x, t)$ and $g_{B}^{\varepsilon}\left(\frac{x_{1}}{\varepsilon}, x_{2}, t\right)$ are lengthy and not important. The smoothness and the compatibility conditions satisfied by $g_{E}^{\varepsilon}$ and $g_{B}^{\varepsilon}$ are

$$
\begin{gathered}
g_{E}^{\varepsilon}(x, t) \in \bigcap_{j=0}^{[m / 2]+1-7 N} C^{j}\left([0, T] ; H^{[m / 2]+1-7 N-j}(\Omega)\right), \\
\partial_{t}^{k} g_{E}^{\varepsilon}(x, 0)=0, \quad \text { for } x \in \Omega, \quad k=0,1,
\end{gathered}
$$

and

$$
\begin{gathered}
g_{B}\left(\varepsilon ; \frac{x_{1}}{\varepsilon}, x_{2}, t\right) \in \bigcap_{j=0}^{[m / 2]-2-7 N} C^{j}\left([0, T] ; H^{[m / 2]-2-7 N-j}(\Omega)\right), \\
\partial_{t}^{k} g_{B}^{\varepsilon}\left(\frac{x_{1}}{\varepsilon}, x_{2}, 0\right)=0, \quad \text { for } \quad x \in \Omega \quad k=0,1, \cdots,\left[\frac{m}{2}\right]-2-7 N .
\end{gathered}
$$

Moreover, it is easy to see that there exists a constant $C$ which is independent of $\varepsilon$, such that

$$
\sup _{t \in[0, T]} \sum_{k+|\alpha| \leq[m / 2]+1-7 N}\left\|\partial_{t}^{k} \partial_{x_{1}}^{\alpha_{1}} \partial_{x_{2}}^{\alpha_{2}} g_{E}^{\varepsilon}(x, t)\right\|_{L^{2}(\Omega)^{2}} \leq C,
$$


and

$$
\sup _{t \in[0, T]} \sum_{k+|\alpha| \leq[m / 2]-2-7 N}\left\|\left\langle z_{1}\right\rangle^{l} \partial_{t}^{k} \partial_{z_{1}}^{\alpha_{1}} \partial_{z_{2}}^{\alpha_{2}} g_{B}^{\varepsilon}(z, t)\right\|_{L^{2}(\Omega)^{2}} \leq C
$$

\section{Estimates of the Error Term of the Approximate Solution}

In this section we estimate the error term of the approximate solution. Let $V^{\varepsilon}$ be the solution of the linearized Navier-Stokes-Fourier equations (1.7), and $W^{\varepsilon}$ be the approximate solution we constructed in the previous sections.

Let

$$
w^{\varepsilon}=V^{\varepsilon}-Q^{-1}(x, t) W^{\varepsilon} \doteq\left(w_{0}^{\varepsilon}, w_{1}^{\varepsilon}, w_{2}^{\varepsilon}, w_{3}^{\varepsilon}\right)^{\top} .
$$

By Proposition 4.1 and the (2.73), we have that

$$
w^{\varepsilon}(x, t) \in \bigcap_{j=0}^{[m / 2]-1-7 N} C^{j}\left([0, T] ; H^{[m / 2]-1-7 N-j}(\Omega)\right) .
$$

We also have that $w^{\varepsilon}$ satisfies the equation

$$
A_{0}\left(V^{\prime}\right) \partial_{t} w^{\varepsilon}+\sum_{j=1}^{2} A_{j}\left(V^{\prime}\right) \partial_{j} w^{\varepsilon}-L_{\varepsilon} w^{\varepsilon}=\varepsilon^{N} G\left(\varepsilon, \frac{x_{1}}{\varepsilon}, x, t\right), \quad(x, t) \in \Omega \times[0, T] .
$$

with the boundary and initial condition

$$
\begin{aligned}
w_{1}^{\varepsilon}=w_{2}^{\varepsilon}=w_{3}^{\varepsilon}=0, & \text { on } \Gamma \times[0, T] \\
w^{\varepsilon}(x, 0)=0, & \text { for } \quad x \in \Omega .
\end{aligned}
$$

Here

$$
\begin{aligned}
G\left(\varepsilon, \frac{x_{1}}{\varepsilon}, x, t\right) & =\varepsilon Q^{-1} g_{E}(\varepsilon, x, t)+Q^{-1} g_{B}\left(\varepsilon, \frac{x_{1}}{\varepsilon}, x_{2}, t\right) \\
& =\left(G_{0}, G_{1}, G_{2}, G_{3}\right)^{\top} .
\end{aligned}
$$

Let $m \geq 2(7 N+4)$, By (2.74) $-(\underline{2.79})$, we have

$$
G\left(\varepsilon ; \frac{x_{1}}{\varepsilon}, x, t\right) \in \bigcap_{j=0}^{2} C^{j}\left([0, T] ; H^{2-j}(\Omega)\right),
$$

and

$$
\partial_{t}^{k} G\left(\varepsilon ; \frac{x_{1}}{\varepsilon}, x, 0\right)=0, \quad k=0,1, \quad \text { for } \quad x \in \Omega
$$

Then Theorem 1.1 is a conclusion of the following proposition:

Proposition 3.1. Assume that $m \geq 2(7 N+4)$, $w^{\varepsilon}$ satisfies (3.81) $-(3.82)$, then we have

$$
w^{\varepsilon} \in \bigcap_{j=0}^{3} C^{j}\left([0, T] ; H^{3-j}(\Omega)\right)
$$

and the following estimates hold

$$
\sup _{(x, t) \in \Omega \times[0, T]}\left|w_{0}^{\varepsilon}\right| \leq C \varepsilon^{N-1}
$$

and

$$
\sup _{(x, t) \in \Omega \times[0, T]}\left|w_{j}^{\varepsilon}\right| \leq C \varepsilon^{N-\frac{3}{4}}, \quad j=1,2,3 .
$$


We write $w=\left(w_{0}, w_{1}, w_{2}, w_{3}\right)^{\top}$ instead of $w^{\varepsilon}$ for simplicity. We rewrite the equation as

$$
\begin{gathered}
\frac{1}{\rho^{\prime}} \partial_{t} w_{0}+\frac{\mathrm{u}^{\prime}}{\rho^{\prime}} \cdot \nabla w_{0}+\left(\partial_{1} w_{1}+\partial_{2} w_{2}\right)=\varepsilon^{N} G_{0} \\
\frac{\rho^{\prime}}{p_{\rho}^{\prime}} \partial_{t} w_{1}+\frac{\rho^{\prime}}{p_{\rho}^{\prime}} \mathrm{u}^{\prime} \cdot \nabla w_{1}+\partial_{1} w_{0}+\frac{p_{\theta}^{\prime}}{p_{\rho}^{\prime}} \partial_{1} w_{3} \\
-\frac{1}{p_{\rho}^{\prime}} \varepsilon^{2}\left(\Delta w_{1}+C \partial_{1}\left(\partial_{1} w_{1}+\partial_{2} w_{2}\right)\right)=\varepsilon^{N} G_{1} \\
\frac{\rho^{\prime}}{p_{\rho}^{\prime}} \partial_{t} w_{2}+\frac{\rho^{\prime}}{p_{\rho}^{\prime}} \mathrm{u}^{\prime} \cdot \nabla w_{2}+\partial_{2} w_{0}+\frac{p_{\theta}^{\prime}}{p_{\rho}^{\prime}} \partial_{2} w_{3} \\
-\frac{1}{p_{\rho}^{\prime}} \varepsilon^{2}\left(\triangle w_{2}+C \partial_{2}\left(\partial_{1} w_{1}+\partial_{2} w_{2}\right)\right)=\varepsilon^{N} G_{2}, \\
\beta^{\prime} \partial_{t} w_{3}+\beta^{\prime} \mathrm{u}^{\prime} \cdot \nabla w_{3}+\frac{p_{\theta}^{\prime}}{p_{\rho}^{\prime}}\left(\partial_{1} w_{1}+\partial_{2} w_{2}\right) \\
-\varepsilon^{2} \frac{\bar{\kappa}}{\theta^{\prime} p_{\rho}^{\prime}} \Delta w_{3}-I(w)=\varepsilon^{N} G_{3},
\end{gathered}
$$

where $\beta^{\prime}=\frac{\rho^{\prime} c_{v}\left(\theta^{\prime}\right)}{\theta^{\prime} p_{\rho}^{\prime}}$ and

$$
I(w)=\varepsilon^{2} \frac{\bar{\kappa}}{\theta^{\prime} p_{\rho}^{\prime}} \Delta w_{3}+\frac{1}{\theta^{\prime} p_{\rho}^{\prime}}\left(\mathbb{S}: \nabla\left(w_{1}, w_{2}\right)^{\top}+\mathbb{S}\left(w_{1}, w_{2}\right)^{\top}: \nabla \mathbf{u}^{\prime}\right) .
$$

In the rest of this section, we will denote $\langle\cdot, \cdot\rangle$ as the inner product in $L^{2}(\Omega)$ and $\|\cdot\|$ as the norm in $L^{2}(\Omega)$, the generic constants $C_{i}, i=1,2, \ldots$ are positive depending only on $V^{\prime}$ and its derivatives. First we derive the basic energy estimate on $w$ :

Lemma 3.1. For any $t \in[0, T]$, we have that

$$
\|w(t)\|^{2}+\varepsilon^{2} \sum_{j=1}^{3} \int_{0}^{t}\left\|\nabla w_{j}\right\|^{2} \mathrm{~d} s \leq C \varepsilon^{2 N+1} .
$$

Proof. Taking the inner product in $L^{2}(\Omega)$ of (3.83)-(3.86) with $w$, by integration by parts we have that:

$$
\begin{aligned}
\frac{1}{2} \frac{\mathrm{d}}{\mathrm{d} t}\left\langle\frac{1}{\rho^{\prime}} w_{0},\right. & \left.w_{0}\right\rangle-\frac{1}{2}\left\langle\left[\partial_{t}\left(\frac{1}{\rho^{\prime}}\right)+\nabla \cdot \frac{u^{\prime}}{\rho^{\prime}}\right] w_{0}, w_{0}\right\rangle-\sum_{j=1}^{2}\left\langle w_{j}+\partial_{j} w_{0}\right\rangle=\left\langle\varepsilon^{N} G_{0}, w_{0}\right\rangle, \\
& \frac{1}{2} \frac{\mathrm{d}}{\mathrm{d} t}\left\langle\frac{\rho^{\prime}}{p_{\rho}^{\prime}} w_{1}, w_{1}\right\rangle-\frac{1}{2}\left\langle\left[\partial_{t}\left(\frac{1}{\rho^{\prime}}\right)+\nabla \cdot \frac{\mathrm{u}^{\prime}}{\rho^{\prime}}\right] w_{1}, w_{1}\right\rangle+\left\langle\partial_{1} w_{0}, w_{1}\right\rangle \\
& +\left\langle\frac{p_{\theta}^{\prime}}{p_{\rho}^{\prime}} \partial_{1} w_{3}, w_{1}\right\rangle+\varepsilon^{2}\left\{\left\langle\frac{1}{p_{\rho}^{\prime}} w_{1}, w_{1}\right\rangle+C\left\langle\frac{1}{p_{\rho}^{\prime}}\left(\partial_{1} w_{1}+\partial_{2} w_{2}\right), \partial_{1} w_{1}\right\rangle\right. \\
& \left.-\left\langle\nabla \frac{1}{p_{\rho}^{\prime}} \cdot \nabla w_{1}\right\rangle-C\left\langle\partial_{1}\left(\frac{1}{p_{\rho}^{\prime}}\right)\left(\partial_{1} w_{1}+\partial_{2} w_{2}\right), w_{1}\right\rangle\right\}=\left\langle\varepsilon^{N} G^{1}, w_{1}\right\rangle \\
& \frac{1}{2} \frac{\mathrm{d}}{\mathrm{d} t}\left\langle\frac{\rho^{\prime}}{p_{\rho}^{\prime}} w_{2}, w_{2}\right\rangle-\frac{1}{2}\left\langle\left[\partial_{t}\left(\frac{1}{\rho^{\prime}}\right)+\nabla \cdot \frac{\mathrm{u}^{\prime}}{\rho^{\prime}}\right] w_{2}, w_{2}\right\rangle+\left\langle\partial_{2} w_{0}, w_{2}\right\rangle \\
& +\left\langle\frac{p_{\theta}^{\prime}}{p_{\rho}^{\prime}} \partial_{2} w_{3}, w_{2}\right\rangle+\varepsilon^{2}\left\{\left\langle\frac{1}{p_{\rho}^{\prime}} w_{2}, w_{2}\right\rangle+C\left\langle\frac{1}{p_{\rho}^{\prime}}\left(\partial_{1} w_{1}+\partial_{2} w_{2}\right), \partial_{2} w_{2}\right\rangle\right. \\
& \left.-\left\langle\nabla \frac{1}{p_{\rho}^{\prime}} \cdot \nabla w_{2}\right\rangle-C\left\langle\partial_{2}\left(\frac{1}{p_{\rho}^{\prime}}\right)\left(\partial_{1} w_{1}+\partial_{2} w_{2}\right), w_{2}\right\rangle\right\}=\left\langle\varepsilon^{N} G^{2}, w_{2}\right\rangle \\
& \frac{1}{2} \frac{\mathrm{d}}{\mathrm{d} t}\left\langle\beta^{\prime} w_{3}, w_{3}\right\rangle-\frac{1}{2}\left\langle\left[\partial_{t} \beta^{\prime}+\nabla \cdot\left(\beta^{\prime} u^{\prime}\right)\right] w_{3}, w_{3}\right\rangle-\left\langle\frac{p_{\theta}^{\prime}}{p_{\rho}^{\prime}} w_{1}, \partial_{1} w_{3}\right\rangle \\
& -\left\langle\frac{p_{\theta}^{\prime}}{p_{\rho}^{\prime}} w_{2}, \partial_{2} w_{3}\right\rangle-\left\langle\nabla \frac{p_{\theta}^{\prime}}{p_{\rho}^{\prime}} \cdot\left(w_{1}, w_{2}\right)^{\top}, w_{3}\right\rangle+\varepsilon^{2}\left\{\left\langle\frac{\kappa_{0}\left(\theta^{\prime}\right)}{\theta^{\prime} p_{\rho}^{\prime}} \nabla w_{3}, \nabla w_{3}\right\rangle\right. \\
& \left.+\left\langle\nabla \frac{\kappa_{0}\left(\theta^{\prime}\right)}{\theta^{\prime} p_{\rho}^{\prime}} w_{3}, \nabla w_{3}\right\rangle\right\}-\left\langle I(w), w_{3}\right\rangle=\varepsilon^{N} G_{3} .
\end{aligned}
$$

Adding the above four equations shows that

$$
\frac{1}{2} \frac{\mathrm{d}}{\mathrm{d} t}\|w\|_{V^{\prime}}^{2}+\varepsilon^{2} \sum_{j=1}^{3}\left\|\nabla w_{j}\right\|^{2} \leq C\left(\|w\|^{2}+\varepsilon^{2 N}\|G\|^{2}\right),
$$


where

and obviously

$$
\|w\|_{V^{\prime}}=\left\langle\frac{1}{\rho^{\prime}} w_{0}, w_{0}\right\rangle+\left\langle\frac{\rho^{\prime}}{p_{\rho}^{\prime}} w_{1}, w_{1}\right\rangle+\left\langle\frac{\rho^{\prime}}{p_{\rho}^{\prime}} w_{2}, w_{2}\right\rangle+\left\langle\beta^{\prime} w_{3}, w_{3}\right\rangle
$$

Then inequality (3.91) reads that

$$
C_{1}\|w\|^{2} \leq\|w\|_{V^{\prime}}^{2} \leq C_{2}\|w\|^{2}
$$

$$
\|w(t)\|^{2}+\varepsilon^{2} \sum_{j=1}^{3} \int_{0}^{t}\left\|\nabla w_{j}\right\|^{2} \leq C\left(\int_{0}^{t}\|w(s)\| \mathrm{d} s+\varepsilon^{2 N} \int_{0}^{t}\|G(\varepsilon, s)\|^{2} \mathrm{~d} s\right) .
$$

It is easy to check that

$$
\|G(\varepsilon, s)\|^{2} \leq C \varepsilon
$$

Then by Gronwall inequality we complete the proof of this lemma.

Next, we will get some estimates of the derivatives of $w$, by the compatibility condition, one could get that

$$
\partial_{t} w_{j}=0, \partial_{2} w_{j}=0, \quad j=1,2, \quad \text { on } \quad \Gamma \times[0, T] .
$$

However, we could not get zero boundary condition for $\partial_{x_{1}} w_{j}$, then we need to define the tangential derivatives of $w$ :

$$
D^{\tan } w=\left(\partial_{t} w, \chi\left(x_{1}\right) \partial_{x_{1}} w, \partial_{x_{2}} w\right)
$$

where $\chi(s) \in C^{3}([0, \infty))$ satisfies

$$
\begin{aligned}
\chi(0) & =0, \quad \chi^{\prime}(0)=1, \\
\chi^{\prime}(s) \geq 0, & \text { for } \quad s \in(0, \infty), \\
\chi(s) & =1, \quad \text { for } \quad s \geq 1 .
\end{aligned}
$$

We have the following estimate:

Lemma 3.2. For any $t \in[0, T]$, we have that

$$
\left\|D^{\tan } w(t)\right\|^{2}+\varepsilon^{2} \sum_{j=1}^{3} \int_{0}^{t}\left\|\nabla D^{\tan } w_{j}(s)\right\|^{2} \mathrm{~d} s \leq C \varepsilon^{2 N-1} .
$$

Proof. Applying $\partial_{t}$ to the equation (3.83)-(3.86), we have that:

$$
\begin{aligned}
& \quad \frac{1}{\rho^{\prime}} \partial_{t}\left(\partial_{t} w_{0}\right)+\frac{\mathrm{u}^{\prime}}{\rho^{\prime}} \cdot \nabla\left(\partial_{t} w_{0}\right)+\left(\partial_{1}\left(\partial_{t} w_{1}\right)+\partial_{2}\left(\partial_{t} w_{2}\right)\right) \\
& \quad+\partial_{t} \frac{1}{\rho^{\prime}} \partial_{t} w_{0}+\partial_{t} \frac{\mathrm{u}^{\prime}}{\rho^{\prime}} \cdot \nabla w_{0}=\varepsilon^{N} \partial_{t} G_{0}, \\
& \frac{\rho^{\prime}}{p_{\rho}^{\prime}} \partial_{t}\left(\partial_{t} w_{1}\right)+\frac{\rho^{\prime}}{p_{\rho}^{\prime}} \mathrm{u}^{\prime} \cdot \nabla\left(\partial_{t} w_{1}\right)+\partial_{1}\left(\partial_{t} w_{0}\right)+\frac{p_{\theta}^{\prime}}{p_{\rho}^{\prime}} \partial_{1}\left(\partial_{t} w_{3}\right) \\
& +\partial_{t}\left(\frac{\rho^{\prime}}{p_{\rho}^{\prime}}\right) \partial_{t} w_{1}+\partial_{t}\left(\frac{\rho^{\prime}}{p_{\rho}^{\prime}} \mathrm{u}^{\prime}\right) \cdot \nabla w_{1}+\partial_{t}\left(\frac{p_{\theta}^{\prime}}{p_{\rho}^{\prime}}\right) \partial_{1} w_{3} \\
& -\varepsilon^{2} \frac{1}{p_{\rho}^{\prime}}\left(\Delta\left(\partial_{t} w_{1}\right)+C \partial_{1}\left(\partial_{1}\left(\partial_{t} w_{1}\right)+\partial_{2}\left(\partial_{t} w_{2}\right)\right)\right) \\
& -\varepsilon^{2} \partial_{t} \frac{1}{p_{\rho}^{\prime}}\left(\Delta w_{1}+C \partial_{1}\left(\partial_{1} w_{1}+\partial_{2} w_{2}\right)\right)=\varepsilon^{N} \partial_{t} G_{1}, \\
& \frac{\rho^{\prime}}{p_{\rho}^{\prime}} \partial_{t}\left(\partial_{t} w_{2}\right)+\frac{\rho^{\prime}}{p_{\rho}^{\prime}} u^{\prime} \cdot \nabla\left(\partial_{t} w_{2}\right)+\partial_{2}\left(\partial_{t} w_{0}\right)+\frac{p_{\theta}^{\prime}}{p_{\rho}^{\prime}} \partial_{2}\left(\partial_{t} w_{3}\right) \\
& +\partial_{t}\left(\frac{\rho^{\prime}}{p_{\rho}^{\prime}}\right) \partial_{t} w_{2}+\partial_{t}\left(\frac{\rho^{\prime}}{p_{\rho}^{\prime}} u^{\prime}\right) \cdot \nabla w_{2}+\partial_{t}\left(\frac{p_{\theta}^{\prime}}{p_{\rho}^{\prime}}\right) \partial_{2} w_{3} \\
& -\varepsilon^{2} \frac{1}{p_{\rho}^{\prime}}\left(\Delta\left(\partial_{t} w_{2}\right)+C \partial_{2}\left(\partial_{1}\left(\partial_{t} w_{1}\right)+\partial_{2}\left(\partial_{t} w_{2}\right)\right)\right) \\
& -\varepsilon^{2} \partial_{t} \frac{1}{p_{\rho}^{\prime}}\left(\Delta w_{2}+C \partial_{2}\left(\partial_{1} w_{1}+\partial_{2} w_{2}\right)\right)=\varepsilon^{N} \partial_{t} G_{2},
\end{aligned}
$$




$$
\begin{aligned}
& \beta^{\prime} \partial_{t}\left(\partial_{t} w_{3}\right)+\beta^{\prime} \mathrm{u}^{\prime} \cdot \nabla\left(\partial_{t} w_{3}\right)+\frac{p_{\theta}^{\prime}}{p_{\rho}^{\prime}}\left(\partial_{1}\left(\partial_{t} w_{1}\right)+\partial_{2}\left(\partial_{t} w_{2}\right)\right) \\
& +\partial_{t} \beta^{\prime} \partial_{t} w_{3}+\partial_{t} \beta^{\prime} \mathrm{u}^{\prime} \cdot \nabla w_{3}+\partial_{t}\left(\frac{p_{\theta}^{\prime}}{p_{\rho}^{\prime}}\right)\left(\partial_{1} w_{1}+\partial_{2} w_{2}\right) \\
& -\frac{\varepsilon^{2} \kappa_{0}\left(\theta^{\prime}\right)}{\theta^{\prime} p_{\rho}^{\prime}} \Delta\left(\partial_{t} w_{3}\right)-\partial_{t} \frac{\varepsilon^{2} \kappa_{0}\left(\theta^{\prime}\right)}{\theta^{\prime} p_{\rho}^{\prime}} \Delta w_{3}-\partial_{t} I(w)=\varepsilon^{N} \partial_{t} G_{3} .
\end{aligned}
$$

Take the inner product in $L^{2}(\Omega)$ of the above equations with $\partial_{t} w$, integrating by parts we have:

$$
\begin{aligned}
& \frac{1}{2} \frac{\mathrm{d}}{\mathrm{d} t}\left\|\partial_{t} w\right\|_{U^{\prime}}^{2}+\varepsilon^{2} \sum_{j=1}^{3}\left\langle\nabla \partial_{t} w_{j}, \nabla \partial_{t} w_{j}\right\rangle+\left\langle\partial_{t}\left(\frac{\rho^{\prime}}{p_{\rho}^{\prime}} \mathrm{u}^{\prime}\right) \cdot w_{0}, \partial_{t} w_{0}\right\rangle \\
& \sum_{j=1}^{2}\left\langle\partial_{t}\left(\frac{\rho^{\prime}}{p_{\rho}^{\prime}} \mathrm{u}^{\prime}\right) \cdot \nabla w_{j}, \partial_{t} w_{j}\right\rangle+\sum_{j=1}^{2}\left\langle\partial_{t}\left(\frac{p_{\theta}^{\prime}}{p_{\rho}^{\prime}}\right) \partial_{j} w_{3}, \partial_{t} w_{j}\right\rangle \\
& +\left\langle\partial_{t}\left(\beta^{\prime} \mathrm{u}^{\prime}\right) \cdot \nabla w_{3}, \partial_{t} w_{3}\right\rangle+\left\langle\partial_{t}\left(\frac{p_{\theta}^{\prime}}{p_{\rho}^{\prime}}\right)\left(\partial_{1} w_{1}+\partial_{2} w_{2}\right), \partial_{t} w_{3}\right\rangle \\
& \leq C\left(\left\|\partial_{t} w\right\|^{2}+\varepsilon^{2 N}\left\|\partial_{t} G\right\|^{2}\right)
\end{aligned}
$$

As $\partial_{t}\left(\frac{u^{\prime}}{\rho^{\prime}}\right)=0$, on $\Gamma \times[0, T]$, we have that

$$
\left\|\partial_{t}\left(\frac{\mathrm{u}^{\prime}}{\rho^{\prime}}\right) \cdot \nabla w_{0}\right\|^{2} \leq C\left\|D^{\tan } w\right\|^{2} .
$$

Integrating (3.97) with respect with $t$, we have that

$$
\begin{aligned}
& \left\|\partial_{t} w(t)\right\|^{2}+\varepsilon^{2} \sum_{j=1}^{3} \int_{0}^{t}\left\|\nabla \partial_{t} w_{j}(s)\right\|^{2} \mathrm{~d} s \\
\leq & C\left\{\int_{0}^{t}\left\|\partial_{t} w(s)\right\|^{2} \mathrm{~d} s+\int_{0}^{t}\left[\sum_{j=1}^{3} \| \nabla w_{j}\left((s)\left\|^{2}+\right\| D^{\tan } w(s) \|^{2}\right]\right\} \mathrm{d} s+\varepsilon^{2 N} \int_{0}^{t}\left\|\partial_{t} G(s)\right\|^{2} \mathrm{~d} s .\right.
\end{aligned}
$$

by Lemma 3.1 we could have that

$$
\left.\left.\left\|\partial_{t} w(t)\right\|^{2}+\varepsilon^{2} \sum_{j=1}^{3} \int_{0}^{t}\left\|\nabla \partial_{t} w_{j}(s)\right\|^{2} \leq C\left[\int_{0}^{t}\left\|D^{\tan } w(s)\right\|^{2}\right]\right\} \mathrm{d} s+\varepsilon^{2 N-1}\right] .
$$

Similarly, apply $\partial_{2}$ to the equation $(\underline{3.83})-(\underline{3.86})$, by energy estimate we could have

$$
\left.\left.\left\|\partial_{2} w(t)\right\|^{2}+\varepsilon^{2} \sum_{j=1}^{3} \int_{0}^{t}\left\|\nabla \partial_{2} w_{j}(s)\right\|^{2} \leq C\left[\int_{0}^{t}\left\|D^{\tan } w(s)\right\|^{2}\right]\right\} d s+\varepsilon^{2 N-1}\right] .
$$

Apply $\chi\left(x_{1}\right) \partial_{1}$ to $(\underline{3.83})-(3.86)$ we have that

$$
\begin{aligned}
& \frac{1}{\rho^{\prime}} \partial_{t}\left(\chi \partial_{1} w_{0}\right)+\frac{\mathrm{u}^{\prime}}{\rho^{\prime}} \cdot \nabla\left(\chi \partial_{1} w_{0}\right)+\left(\partial_{1}\left(\chi \partial_{1} w_{0}\right)+\partial_{2}\left(\chi \partial_{1} w_{0}\right)\right) \\
& -\frac{\mathrm{u}^{\prime}}{\rho^{\prime}} \chi^{\prime} \partial_{1} w_{0}-\chi^{\prime} \partial_{1} w_{1}+\chi \partial_{1}\left(\frac{1}{\rho^{\prime}}\right) \partial_{t} w_{0}+\chi \mid p a_{1}\left(\frac{\mathbf{u}^{\prime}}{\rho^{\prime}}\right) \cdot \nabla w_{0} \\
& =\varepsilon^{N} \chi \partial_{1} G_{0},
\end{aligned}
$$




$$
\begin{aligned}
& \frac{\rho^{\prime}}{p_{\rho}^{\prime}} \partial_{t}\left(\chi \partial_{1} w_{1}\right)+\frac{\rho^{\prime}}{p_{\rho}^{\prime}} \mathrm{u}^{\prime} \cdot \nabla\left(\chi \partial_{1} w_{1}\right)+\partial_{1}\left(\chi \partial_{1} w_{0}\right)+\frac{p_{\theta}^{\prime}}{p_{\rho}^{\prime}} \partial_{1}\left(\chi \partial_{1} w_{3}\right) \\
& -\frac{\rho^{\prime}}{p_{\rho}^{\prime}} \mathrm{u}_{1}^{\prime} \partial_{1} w_{1}-\chi^{\prime} \partial_{1} w_{0}-\frac{p_{\theta}^{\prime}}{p_{\rho}^{\prime}} \chi^{\prime} \partial_{1} w_{3}+\chi \partial_{1}\left(\frac{\rho^{\prime}}{p_{\rho}^{\prime}}\right) \partial_{t} w_{1} \\
& +\chi \partial_{1}\left(\frac{\rho^{\prime}}{p_{\rho}^{\prime}} \mathrm{u}^{\prime}\right) \cdot \nabla w_{1}+\chi \partial_{1}\left(\frac{p_{\theta}^{\prime}}{p_{\rho}^{\prime}}\right) \partial_{1} w_{3}-\frac{1}{p_{\rho}^{\prime}} \varepsilon^{2}\left(\Delta\left(\chi \partial_{1} w_{1}\right)\right. \\
& \left.+C \partial_{1}\left(\partial_{1}\left(\chi \partial_{1} w_{1}\right)+\partial_{2}\left(\chi \partial_{1} w_{1}\right)\right)\right)+\frac{1+C}{p_{\rho}^{\prime}} \varepsilon^{2}\left(\chi^{\prime \prime} \partial_{1} w_{1}+2 \chi^{\prime} \partial_{1}^{2} w_{1}\right) \\
& +\frac{C}{p_{\rho}^{\prime}} \varepsilon^{2} \chi^{\prime} \partial_{12}^{2} w_{2}-\chi \varepsilon^{2} \partial_{1}\left(\frac{1}{p_{\rho}^{\prime}}\right)\left(\Delta w_{1}+C \partial_{1}\left(\partial_{1} w_{1}+\partial_{2} w_{2}\right)\right)=\varepsilon^{N} \chi \partial_{1} G_{1}, \\
& \frac{\rho^{\prime}}{p_{\rho}^{\prime}} \partial_{t}\left(\chi \partial_{1} w_{2}\right)+\frac{\rho^{\prime}}{p_{\rho}^{\prime}} \mathrm{u}^{\prime} \cdot \nabla\left(\chi \partial_{1} w_{2}\right)+\partial_{2}\left(\chi \partial_{1} w_{0}\right)+\frac{p_{\theta}^{\prime}}{p_{\rho}^{\prime}} \partial_{2}\left(\chi \partial_{1} w_{3}\right) \\
& -\frac{\rho^{\prime}}{p_{\rho}^{\prime}} \mathrm{u}_{1}^{\prime} \partial_{1} w_{2}-\frac{p_{\theta}^{\prime}}{p_{\rho}^{\prime}} \chi^{\prime} \partial_{2} w_{3}+\chi \partial_{1}\left(\frac{\rho^{\prime}}{p_{\rho}^{\prime}}\right) \partial_{t} w_{2}+\chi \partial_{1}\left(\frac{\rho^{\prime}}{p_{\rho}^{\prime}} \mathrm{u}^{\prime}\right) \cdot \nabla w_{2} \\
& +\chi \partial_{1}\left(\frac{p_{\theta}^{\prime}}{p_{\rho}^{\prime}}\right) \partial_{2} w_{3}-\frac{1}{p_{\rho}^{\prime}} \varepsilon^{2}\left(\Delta\left(\chi \partial_{1} w_{2}\right)+C \partial_{2}\left(\partial_{1}\left(\chi \partial_{1} w_{1}\right)+\partial_{2}\left(\chi \partial_{1} w_{1}\right)\right)\right) \\
& +\frac{1}{p_{\rho}^{\prime}} \varepsilon^{2}\left(\chi^{\prime \prime} \partial_{1} w_{2}+2 \chi^{\prime} \partial_{1}^{2} w_{2}\right)+\frac{C}{p_{\rho}^{\prime}} \varepsilon^{2} \chi^{\prime} \partial_{12}^{2} w_{1} \\
& -\chi \varepsilon^{2} \partial_{1}\left(\frac{1}{p_{\rho}^{\prime}}\right)\left(\Delta w_{2}+C \partial_{2}\left(\partial_{1} w_{1}+\partial_{2} w_{2}\right)\right)=\varepsilon^{N} \chi \partial_{1} G_{2}, \\
& \beta^{\prime} \partial_{t}\left(\chi \partial_{1} w_{3}\right)+\beta^{\prime} u^{\prime} \cdot \nabla\left(\chi \partial_{1} w_{3}\right)+\frac{p_{\theta}^{\prime}}{p_{\rho}^{\prime}}\left(\partial_{1}\left(\chi \partial_{1} w_{1}\right)+\partial_{2}\left(\chi \partial_{1} w_{2}\right)\right) \\
& -\beta^{\prime} u^{\prime} \chi^{\prime} \partial_{1} w_{3}-\frac{p_{\theta}^{\prime}}{p_{\rho}^{\prime}} \chi^{\prime} \partial_{1} w_{1}+\chi \partial_{1} \beta^{\prime} \partial_{t} w_{3}+\chi \partial_{1}\left(\beta^{\prime} u^{\prime}\right) \cdot \nabla w_{3} \\
& +\chi \partial_{1}\left(\frac{p_{\theta}^{\prime}}{p_{\rho}^{\prime}}\right)\left(\partial_{1} w_{1}+\partial_{2} w_{2}\right)-\frac{\varepsilon^{2} \kappa_{0}\left(\theta^{\prime}\right)}{\theta^{\prime} p_{\rho}^{\prime}} \Delta\left(\chi \partial_{1} w_{3}\right)+\frac{\varepsilon^{2} \kappa_{0}\left(\theta^{\prime}\right)}{\theta^{\prime} p_{\rho}^{\prime}} \chi^{\prime \prime} \partial_{1} w_{3} \\
& +\frac{2 \varepsilon^{2} \kappa_{0}\left(\theta^{\prime}\right)}{\theta^{\prime} p_{\rho}^{\prime}} \chi^{\prime} \partial_{1}^{2} w_{3}-\partial_{1}\left(\frac{\varepsilon^{2} \kappa_{0}\left(\theta^{\prime}\right)}{\theta^{\prime} p_{\rho}^{\prime}}\right) \chi \Delta w_{3}-\chi \partial_{1} I(w)=\varepsilon^{N} \chi \partial_{1} G_{3},
\end{aligned}
$$

Since $u_{1}^{\prime}=0$ on $\Gamma \times[0, T]$, we have that

$$
\left\langle\frac{\mathrm{u}_{1}^{\prime}}{\rho^{\prime}} \chi^{\prime} \partial_{1} w_{0}, \chi \partial_{1} w_{0}\right\rangle \leq C\left\|\chi \partial_{1} w_{0}\right\|^{2}
$$

and we also have that

$$
\left\langle\frac{\chi^{\prime}}{p_{\rho}^{\prime}} \partial_{11} w_{1}, \chi \partial_{1} w_{1}\right\rangle=-\frac{1}{2}\left(\left\langle\partial_{1}\left(\frac{\chi^{\prime}}{p_{\rho}^{\prime}}\right) \partial_{1} w_{1}, \chi \partial_{1} w_{1}\right\rangle-\left\langle\frac{\chi^{\prime}}{p_{\rho}^{\prime}} \partial_{1} w_{1}, \chi^{\prime} \partial_{1} w_{1}\right\rangle\right)
$$

and

$$
\begin{aligned}
& \int_{\Omega}\left|\chi\left(x_{1}\right) \partial_{x_{1}} g_{B}\left(\frac{x_{1}}{\varepsilon}, x_{2}, t\right)\right|^{2} \mathrm{~d} x_{1} \mathrm{~d} x_{2} \\
= & \varepsilon \int_{\Omega}\left|\frac{\chi\left(\varepsilon z_{1}\right)}{\varepsilon} \partial_{z_{1}} g_{B}\left(z_{1}, x_{2}, t\right)\right|^{2} \mathrm{~d} z_{1} \mathrm{~d} x_{2} \\
\leq & C \varepsilon .
\end{aligned}
$$

Let us take the inner product in $L^{2}(\Omega)$ of (3.100) $)$-(3.103) with $\chi \partial_{1} w_{1}$, integrating with respect to $t$. Then by (3.104), (3.105) ) and (3.106) we could get that

$$
\begin{aligned}
& \left\|\chi\left(x_{1}\right) \partial_{1} w(t)\right\|^{2}+\varepsilon^{2} \sum_{j=1}^{3} \int_{0}^{t}\left\|\nabla\left(\chi \partial_{1} w_{j}(s)\right)\right\|^{2} \mathrm{~d} s \\
\leq & C\left(\int_{0}^{t}\left\|D^{\tan } w(s)\right\|^{2} d s+\varepsilon^{2 N-1}\right) .
\end{aligned}
$$

Collecting the estimates (3.98) $)-(3.99)$ and (3.107), we complete the proof of this lemma. 
Lemma 3.3. For all $t \in[0, T]$, we have the following estimate

$$
\sum_{j=1}^{3}\left\|\partial_{1} w_{j}(t)\right\|^{2} \leq C \varepsilon^{2 N} .
$$

Proof. By estimate (3.91), we have that

$$
\begin{aligned}
\varepsilon^{2} \sum_{j=1}^{3}\left\|\partial_{1} w_{j}(t)\right\|^{2} & \leq\left|\frac{1}{2} \frac{\mathrm{d}}{\mathrm{d} t}\|w(t)\|_{U^{\prime}}^{2}\right|+C_{2}\left(\|w(t)\|^{2}+\varepsilon^{2 N}\|G\|^{2}\right) \\
& \leq C\left\{\varepsilon\left\|\partial_{t} w(t)\right\|^{2}+\frac{1}{\varepsilon}\|w(t)\|^{2}+\|w(t)\|^{2}+\varepsilon^{2 N+1}\right\}
\end{aligned}
$$

by Lemma 3.1 and Lemma 3.2 we have that

$$
\|w(t)\|^{2} \leq C \varepsilon^{2 N+1}
$$

and

$$
\left\|\partial_{t} w(t)\right\|^{2} \leq C \varepsilon^{2 N-1} .
$$

then we complete the proof of this lemma.

Lemma 3.4. For any $t \in[0, T]$, the following estimate holds

$$
\varepsilon^{2}\left\|\partial_{1} w_{0}(t)\right\|^{2}+\varepsilon \int_{0}^{t}\left\|\partial_{1} w_{0}(s)\right\|^{2} \mathrm{~d} s \leq C \varepsilon^{2 N-1} .
$$

Proof. Apply $\partial_{1}$ to the equation (3.83) and we get that

$$
\begin{aligned}
& \frac{1}{\rho^{\prime}} \partial_{t}\left(\partial_{1} w_{0}\right)+\frac{\mathrm{u}^{\prime}}{\rho^{\prime}} \cdot \nabla\left(\partial_{1} w_{0}\right)+\partial_{1}\left(\frac{1}{\rho^{\prime}} \mathrm{u}^{\prime}\right) \cdot \nabla w_{0}+\partial_{11} w_{1}+\partial_{12} w_{2} \\
= & \varepsilon^{N} \partial_{1} G_{0},
\end{aligned}
$$

by (3.84), we have that

$$
\begin{aligned}
\partial_{11} w_{1}= & \frac{1}{\varepsilon^{2}(1+C)}\left(\rho^{\prime} \partial_{t} w_{1}+\rho^{\prime} u^{\prime} \cdot \nabla w_{1}\right. \\
& \left.+p_{\rho}^{\prime} \partial_{1} w_{0}+p_{\theta}^{\prime} \partial_{1} w_{3}-C \partial_{12} w_{2}-p_{\rho}^{\prime} \varepsilon^{N} G_{1}\right) .
\end{aligned}
$$

Thus we could eliminate $\partial_{11} w_{1}$ from (3.109), and take the inner product in $L^{2}(\Omega)$ of this equality with $\partial_{1} w_{0}(t)$, and integrating with respect to $t$, we obtain

$$
\begin{aligned}
& \left\|\partial_{1} w_{0}(t)\right\|^{2}+\frac{1}{\varepsilon^{2}} \int_{0}^{t}\left\|\partial_{1} w_{0}(s)\right\|^{2} \mathrm{~d} s \\
\leq & C\left(\int_{0}^{t}\left\|\partial_{1} w_{0}(s)\right\|^{2} d s+\frac{1}{\varepsilon^{2}} \int_{0}^{t} \sum_{j=1}^{3}\left\|D^{\tan } w_{j}(s)\right\|^{2} \mathrm{~d} s+\varepsilon^{2 N-1}\right)
\end{aligned}
$$

by Lemma 3.2, we deduce that

$$
\left\|\partial_{1} w_{0}(t)\right\|^{2}+\frac{1}{\varepsilon^{2}} \int_{0}^{t}\left\|\partial_{1} w_{0}(t)\right\|^{2} d s \leq C \varepsilon^{2 N-3}
$$

then we complete the proof of this lemma.

Next, we will derive some estimates of higher-order derivatives of $w$. 
Lemma 3.5. For any $t \in[0, T]$, we have that

$$
\begin{aligned}
& \left\|\partial_{22} w(t)\right\|^{2}+\left\|\chi \partial_{12} w(t)\right\|^{2}+\left\|\chi \partial_{11} w(t)\right\|^{2}+\int_{0}^{t} \sum_{j=1}^{2}\left\|\chi \partial_{t j} w_{0}(s)\right\|^{2} \mathrm{~d} s \\
& +\varepsilon^{2} \sum_{j=1}^{3} \int_{0}^{t}\left(\left\|\nabla\left(\partial_{22} w_{j}(s)\right)\right\|^{2}+\left\|\chi \nabla\left(\partial_{12} w_{j}(s)\right)\right\|^{2}+\left\|\nabla\left(\chi \partial_{11} w_{j}(s)\right)\right\|^{2}\right. \\
& \left.+\left\|\partial_{11} w_{j}(s)\right\|^{2}\right) \mathrm{d} s \leq C \varepsilon^{2 N-3} .
\end{aligned}
$$

Proof. First, apply $\partial_{22}$ to the equation (3.83)-(3.86), and take the inner product in $L^{2}(\Omega)$ with $\partial_{22} w$, we could get that

$$
\begin{aligned}
& \left\|\partial_{22} w(t)\right\|^{2}+\varepsilon^{2} \sum_{j=1}^{3} \int_{0}^{t}\left\|\nabla \partial_{22} w_{j}(s)\right\|^{2} \mathrm{~d} s \\
\leq & C\left(\int_{0}^{t}\left(\left\|\partial_{22} w_{0}(s)\right\|^{2}+\left\|\partial_{t x_{2}} w_{0}(s)\right\|^{2}+\left\|\partial_{2}\left(\chi \partial_{1} w_{0}(s)\right)\right\|^{2}\right) \mathrm{d} s+\varepsilon^{2 N-3}\right) .
\end{aligned}
$$

Applying $\partial_{2}$ to the equation (3.100)-(3.103), and taking the inner product in $L^{2}(\Omega)$ with $\partial_{2}\left(\chi \partial_{1} w\right)$, we could get that

$$
\begin{aligned}
& \left\|\partial_{2}\left(\chi \partial_{1} w(t)\right)\right\|^{2}+\varepsilon^{2} \sum_{j=1}^{3} \int_{0}^{t}\left\|\nabla \partial_{2}\left(\chi \partial_{1} w_{j}(s)\right)\right\|^{2} d s \\
\leq & C\left(\int _ { 0 } ^ { t } \left[\left\|\partial_{2}\left(\chi \partial_{1} w(s)\right)\right\|^{2}+\left\|\partial_{22} w_{0}(s)\right\|^{2}+\left\|\chi \partial_{11} w_{0}(s)\right\|^{2}\right.\right. \\
& \left.\left.+\left\|\partial_{t x_{2}} w_{0}(s)\right\|^{2}+\left\|\partial_{t}\left(\chi \partial_{1} w_{0}(s)\right)\right\|^{2}\right] d s+\varepsilon^{2 N-3}\right),
\end{aligned}
$$

take inner product in $L^{2}(\Omega)$ of (3.100) with $\chi \partial_{1} w_{0}$, we derive

$$
\int_{0}^{t}\left\|\partial_{t}\left(\chi \partial_{1} w_{0}(s)\right)\right\|^{2} \mathrm{~d} s \leq C\left\{\int_{0}^{t}\left[\left\|\partial_{2}\left(\chi \partial_{1} w_{0}(s)\right)\right\|^{2}+\left\|\chi \partial_{11} w_{0}\right\|^{2}\right] d s+\varepsilon^{2 N-3}\right\} .
$$

By the same way, applying $\partial_{2}$ to (3.83) and taking inner product in $L^{2}(\Omega)$ with $\partial_{2} w_{0}$, we have that

$$
\int_{0}^{t}\left\|\partial_{t}\left(\partial_{2} w_{0}(s)\right)\right\|^{2} \mathrm{~d} s \leq C\left\{\int_{0}^{t}\left[\left\|\partial_{2}\left(\chi \partial_{1} w_{0}(s)\right)\right\|^{2}+\left\|\chi \partial_{22} w_{0}\right\|^{2}\right] d s+\varepsilon^{2 N-3}\right\} .
$$

Plugging (3.115) and (3.116) into (3.114), we obtain that

$$
\begin{aligned}
& \left\|\partial_{2}\left(\chi \partial_{1} w(t)\right)\right\|^{2}+\varepsilon^{2} \sum_{j=1}^{3} \int_{0}^{t}\left\|\nabla \partial_{2}\left(\chi \partial_{1} w_{j}(s)\right)\right\|^{2} \mathrm{~d} s \\
\leq & C\left(\int_{0}^{t}\left[\left\|\partial_{2}\left(\chi \partial_{1} w(s)\right)\right\|^{2}+\left\|\partial_{22} w_{0}(s)\right\|^{2}+\left\|\chi \partial_{11} w_{0}(s)\right\|^{2}\right] \mathrm{d} s+\varepsilon^{2 N-3}\right) .
\end{aligned}
$$

Also by (3.113) and (3.116) we get

$$
\begin{aligned}
& \left\|\partial_{22} w(t)\right\|^{2}+\varepsilon^{2} \sum_{j=1}^{3} \int_{0}^{t}\left\|\nabla \partial_{22} w_{j}(s)\right\|^{2} \mathrm{~d} s \\
\leq & C\left(\int_{0}^{t}\left(\left\|\partial_{22} w_{0}(s)\right\|^{2}+\left\|\partial_{2}\left(\chi \partial_{1} w_{0}(s)\right)\right\|^{2}\right) \mathrm{d} s+\varepsilon^{2 N-3}\right) .
\end{aligned}
$$


Next, applying $\chi \partial_{11}$ to the equation (3.83)-(3.86) and taking inner product in $L^{2}(\Omega)$ with $\chi \partial_{11} w$, then we could get that

$$
\begin{gathered}
\left\|\chi \partial_{11} w(t)\right\|^{2}+\varepsilon^{2} \sum_{j=1}^{3} \int_{0}^{t}\left\|\nabla\left(\chi \partial_{11} w_{j}(s)\right)\right\|^{2} \mathrm{~d} s \\
\left.\leq C\left(\int_{0}^{t}\left[\| \chi \partial_{11} w(s)\right)\left\|^{2}+\right\| \partial_{2}\left(\chi \partial_{1} w_{0}(s)\right)\left\|^{2}+\varepsilon^{2} \sum_{j=1}^{3}\right\| \partial_{11} w_{j}(s) \|^{2}\right] \mathrm{d} s+\varepsilon^{2 N-3}\right) .
\end{gathered}
$$

From (3.110), we could get that

$$
\begin{aligned}
& \int_{0}^{t}\left\|\partial_{11} w_{1}(s)\right\|^{2} \mathrm{~d} s \\
\leq & C\left\{\frac{1}{\varepsilon^{4}} \int_{0}^{t}\left(\left\|D^{\tan } w_{1}(s)\right\|^{2}+\left\|\partial_{1} w_{0}(s)\right\|^{2}\right) \mathrm{d} s\right. \\
& \left.+\int_{0}^{t}\left(\left\|\partial_{22} w_{1}(s)\right\|^{2}+\left\|\partial_{12} w_{2}(s)\right\|^{2}\right) d s+\varepsilon^{2 N-4} \int_{0}^{t}\|G(s)\|^{2} \mathrm{~d} s\right\} \\
\leq & C \varepsilon^{2 N-5},
\end{aligned}
$$

Similarly, we could also have

$$
\int_{0}^{t}\left\|\partial_{11} w_{j}(s)\right\|^{2} \mathrm{~d} s \leq C \varepsilon^{2 N-5}, \quad \text { for } \quad j=2,3 .
$$

Plugging (3.120) and (3.121) into (3.119), we obtain that

$$
\begin{aligned}
& \left\|\chi \partial_{11} w(t)\right\|^{2}+\varepsilon^{2} \sum_{j=1}^{3} \int_{0}^{t}\left\|\nabla\left(\chi \partial_{11} w_{j}(s)\right)\right\|^{2} \mathrm{~d} s \\
\leq & \left.C\left(\int_{0}^{t}\left[\| \chi \partial_{11} w(s)\right)\left\|^{2}+\right\| \partial_{2}\left(\chi \partial_{1} w_{0}(s)\right) \|^{2}\right] \mathrm{d} s+\varepsilon^{2 N-3}\right) .
\end{aligned}
$$

Collecting the estimates (3.118), (3.120), (3.121) and (3.122), we finally complete the proof of this lemma.

Lemma 3.6. For any $t \in[0, T]$, we have that

$$
\left\|\partial_{12} w_{0}(t)\right\|^{2} \leq C \varepsilon^{2 N-5} .
$$

Proof. Applying $\partial_{12}$ to (3.83), taking the inner product in $L^{2}(\Omega)$ with $\partial_{12} w_{0}$, one can show that

$$
\begin{aligned}
\left\|\partial_{12} w_{0}(t)\right\|^{2} \leq & C\left\{\int _ { 0 } ^ { t } \left(\left\|\partial_{12} w_{0}(s)\right\|^{2}+\left\|\partial_{t x_{1}} w_{0}(s)\right\|^{2}\right.\right. \\
& \left.\left.-\sum_{j=1}^{2}\left\langle\partial_{12} w_{0}, \partial_{j}\left(\partial_{12} w_{j}\right)\right\rangle(s)\right) \mathrm{d} s+\varepsilon^{2 N-3}\right\}
\end{aligned}
$$

Applying $\partial_{1}$ to (3.83), one could show that

$$
\int_{0}^{t}\left\|\partial_{t x_{1}} w_{0}(s)\right\|^{2} \mathrm{~d} s \leq C\left(\int_{0}^{t}\left\|\partial_{12} w_{0}(s)\right\|^{2} d s+\varepsilon^{2 N-5}\right),
$$

Furthermore, by (3.110) we have that

$$
\begin{aligned}
& -\left\langle\partial_{12} w_{0}, \partial_{1}\left(\partial_{12} w_{1}\right)\right\rangle=-\left\langle\partial_{12} w_{0}, \partial_{2}\left(\partial_{11} w_{1}\right)\right\rangle \\
\leq & -\frac{C}{(1+C) \varepsilon^{2}}\left\|\partial_{12} w_{0}\right\|^{2}+\frac{C}{\varepsilon^{2}}\left[\left\|\chi \partial_{12} w_{1}\right\|^{2}+\left\|\partial_{22} w_{1}\right\|^{2}+\left\|D^{\tan } w_{1}\right\|^{2}\right. \\
& \left.+\varepsilon^{2 N}\left\|\partial_{2} G_{1}\right\|^{2}+\varepsilon^{4}\left\|\partial_{x_{2}}^{3} w_{1}\right\|^{2}+\varepsilon^{4}\left\|\partial_{x_{1}} \partial_{x_{2}}^{2} w_{2}\right\|^{2}\right] .
\end{aligned}
$$


Thus by Lemma 3.3, Lemma 3.4 and Lemma 3.5 we have

$$
\begin{aligned}
& -\int_{0}^{t}\left\langle\partial_{12} w_{0}, \partial_{1}\left(\partial_{12} w_{1}\right)\right\rangle(s) \mathrm{d} s \\
\leq & -\frac{C}{(1+C) \varepsilon^{2}} \int_{0}^{t}\left\|\partial_{12} w_{0}(s)\right\|^{2} \mathrm{~d} s+C \varepsilon^{2 N-5},
\end{aligned}
$$

we also have that

$$
\begin{aligned}
& -\int_{0}^{t}\left\langle\partial_{12} w_{0}, \partial_{1}\left(\partial_{22} w_{2}\right)\right\rangle(s) d s \\
\leq & \frac{C}{2(1+C) \varepsilon^{2}} \int_{0}^{t}\left\|\partial_{12} w_{0}(s)\right\|^{2} \mathrm{~d} s+C \varepsilon^{2} \int_{0}^{t}\left\|\partial_{1}\left(\partial_{22} w_{2}(s)\right)\right\|^{2} \mathrm{~d} s \\
\leq & \frac{C}{2(1+C) \varepsilon^{2}} \int_{0}^{t}\left\|\partial_{12} w_{0}(s)\right\|^{2} \mathrm{~d} s+C \varepsilon^{2 N-3} .
\end{aligned}
$$

Collecting the estimates (3.123)-(3.127), we have that

$$
\left\|\partial_{12} w_{0}(t)\right\|^{2} \leq C\left\{\int_{0}^{t}\left\|\partial_{12} w_{0}(s)\right\|^{2} \mathrm{~d} s+\varepsilon^{2 N-5}\right\},
$$

and finally complete the proof of this lemma.

Lemma 3.7. For any $t \in[0, T]$, we have that

$$
\left\|\partial_{t x_{2}} w(t)\right\|^{2}+\left\|\partial_{t t} w(t)\right\|^{2} \leq C \varepsilon^{2 N-3} .
$$

Proof. Applying $\partial_{t x_{2}}$ to (3.83)-(3.86) , taking the inner product in $L^{2}(\Omega)$ with $\partial_{t x_{2}} w$, one can show that

$$
\begin{gathered}
\left\|\partial_{t x_{2}} w(t)\right\|^{2}+\varepsilon^{2} \sum_{j=1}^{3} \int_{0}^{t}\left\|\nabla \partial_{t x_{2}} w_{j}(s)\right\|^{2} \mathrm{~d} s \\
\leq C\left(\int_{0}^{t}\left(\left\|\partial_{t x_{2}} w(s)\right\|^{2}+\left\|\partial_{t t} w(s)\right\|^{2}\right) \mathrm{d} s+\varepsilon^{2 N-3}\right)
\end{gathered}
$$

Similarly, we have that

$$
\begin{gathered}
\left\|\partial_{t t} w(t)\right\|^{2}+\varepsilon^{2} \sum_{j=1}^{3} \int_{0}^{t}\left\|\nabla \partial_{t t} w_{j}(s)\right\|^{2} \mathrm{~d} s \\
\leq C\left(\int_{0}^{t}\left(\left\|\partial_{t x_{2}} w(s)\right\|^{2}+\left\|\partial_{t t} w(s)\right\|^{2}\right) \mathrm{d} s+\varepsilon^{2 N-3}\right) .
\end{gathered}
$$

Combining the above two inequality, we proved the lemma .

Lemma 3.8. For any $t \in[0, T]$, we have that

$$
\sum_{j=1}^{3}\left\|\nabla \partial_{2} w_{j}(t)\right\|^{2} \leq C \varepsilon^{2 N-4} .
$$

Proof. Applying $\partial_{2}$ to (3.83)-(3.86) , taking the inner product in $L^{2}(\Omega)$ with $\partial_{2} w$, by the above lemmas, one can show that

$$
\frac{1}{2} \frac{\mathrm{d}}{\mathrm{d} t}\left\|\partial_{2} w(t)\right\|^{2}+\varepsilon^{2} \sum_{j=1}^{3}\left\|\nabla \partial_{2} w_{j}(t)\right\|^{2} \leq C \varepsilon^{2 N-2} .
$$


Thus

$$
\begin{aligned}
& \varepsilon^{2} \sum_{j=1}^{3}\left\|\nabla \partial_{2} w_{j}(t)\right\|^{2} \\
\leq & -\frac{1}{2} \frac{\mathrm{d}}{\mathrm{d} t}\left\|\partial_{2} w(t)\right\|^{2}+C \varepsilon^{2 N-2} \\
\leq & \sum_{j=0}^{3}\left\|\partial_{2} w(t)\right\|\left\|\partial_{t x_{2}} w(t)\right\|+C_{44} \varepsilon^{2 N-2} \\
\leq & \frac{1}{\varepsilon}\left\|\partial_{2} w(t)\right\|^{2}+\varepsilon\left\|\partial_{t x_{2}} w(t)\right\|^{2}+C \varepsilon^{2 N-2} \\
\leq & C \varepsilon^{2 N-2}
\end{aligned}
$$

To get the $L^{\infty}$ norm estimate of $w$, we need the following proposition which has been also used in [8]:

Proposition 3.2. Let $f\left(x_{1}, x_{2}\right) \in H^{1}(\Omega)$, and $\partial_{12} f \in L^{2}(\Omega)$. Then we have

$$
\left\|f\left(x_{1}, x_{2}\right)\right\|_{L^{\infty}(\Omega)} \leq 2\|f\|^{\frac{1}{4}}\left\|\partial_{1} f\right\|^{\frac{1}{4}}\left\|\partial_{2} f\right\|^{\frac{1}{4}}\left\|\partial_{12} f\right\|^{\frac{1}{4}} .
$$

Based on the above estimates and this proposition, we established proposition 3.1 .

\section{Linear System of Prandtl-type Equations}

In this section, we collect results on the existence on the initial boundary problems for linearized Navier-Stokes-Fourier equations, linearized Euler equations and the linear system of Prandtl-type equations which are frequently used in the previous sections.

First, for the problem (1.7) of the linearized Navier-Stokes equations of a compressible viscous fluid for fixed $\varepsilon$, one can show the following result by the similar argument in [3] with suitable modifications. It was also stated in [8] (see Proposition 1.3 in [8]).

Proposition 4.1. Let $\varepsilon>0$ and let $m>2$ be an integer. Assume that $V_{0} \in H^{m}(\Omega)$ satisfies the compatibility condition of order $[\mathrm{m} / 2]-1$ for initial boundary problem (1.7). Then there exist a unique solution $V$ of (1.7) satisfying

$$
V \in \bigcap_{j=0}^{[m / 2]} C^{j}\left([0, T] ; H^{m-2 j}(\Omega)\right) .
$$

We now state some result of the following linearized Euler equations with inhomogeneous source term

$$
\begin{aligned}
\mathcal{A}_{0} \partial_{t} E-\mathcal{L} E=F, & \text { in } \Omega \times[0, T], \\
\mathcal{M}^{0} E=0, & \text { on } \Gamma \times[0, T], \\
E(x, 0)=f(x), & \text { for } \quad x \in \Omega .
\end{aligned}
$$

As explained in [8], by modifying the argument in [6], we have the following existence of the initial value problem (4.133):

Proposition 4.2. Let $m$ be an integer, and assume that $f(x) \in H^{m}(\Omega)$ and $F(x, t) \in H^{m}(\Omega \times$ $[0, T])$ satisfy the compatibility condition of $m-1$. Then there exists a unique solution to (4.133)

$$
E \in \bigcap_{j=0}^{m} C^{j}\left([0, T] ; H^{m-j}(\Omega)\right)
$$


In the rest of this section, we shall prove the property of the linear system of the Prandtl-type equations. The notation of of the following part of this section is different from that of the other sections. The initial boundary value problem of linear system of the Prandtl-type equations can be written as

$$
\begin{gathered}
\quad a_{1}\left(0, z_{2}, t\right) \partial_{t} u_{1}+b_{11}\left(0, z_{2}, t\right) z_{1} \partial_{z_{1}} u_{1}+b_{12}\left(0, z_{2}, t\right) z_{1} \partial_{z_{1}} u_{2} \\
+c_{11}\left(0, z_{2}, t\right) u_{1}+c_{12}\left(0, z_{2}, t\right) u_{2}-\partial_{z_{1}}^{2} u_{1}=f_{1}\left(z_{1}, z_{2}, t\right), \\
a_{2}\left(0, z_{2}, t\right) \partial_{t} u_{2}+b_{21}\left(0, z_{2}, t\right) z_{1} \partial_{z_{1}} u_{1}+b_{22}\left(0, z_{2}, t\right) z_{1} \partial_{z_{1}} u_{2} \\
+c_{21}\left(0, z_{2}, t\right) u_{1}+c_{22}\left(0, z_{2}, t\right) u_{2}-\partial_{z_{1}}^{2} u_{2}=f_{2}\left(z_{1}, z_{2}, t\right),
\end{gathered}
$$

for $\left(z_{1}, z_{2}, t\right) \in \Omega \times[0, T]$. The boundary condition and initial condition are

$$
\begin{gathered}
u_{1}=u_{2}=0, \quad \text { on } \Gamma \times[0, T], \\
\left(u_{1}, u_{2}\right)\left(z_{1}, z_{2}, 0\right)=(0,0), \quad \text { for } z \in \Omega,
\end{gathered}
$$

where $\Omega=\mathbb{R}_{+}^{2}=\left\{z=\left(z_{1}, z_{2}\right) \in \mathbb{R}^{2}: z_{1} \geq 0\right\}, \Gamma=\partial \Omega$. It is assumed that

$$
a_{i}, b_{i j}, c_{i j} \in C^{\infty}(\Gamma \times[0, T]),
$$

and

$$
a_{i} \geq c_{0}>0 \text { on } \Gamma \times[0, T]
$$

for $i, j=1,2$, Let $m$ and $s$ be integers such that $m \geq s$. $\left(f_{1}, f_{2}\right)$ is assumed to satisfy the following condition:

$$
\left\langle z_{1}\right\rangle^{l} \partial_{t}^{k} \partial_{z_{1}}^{\alpha_{1}} \partial_{z_{2}}^{\alpha_{2}} f_{i} \in C^{0}\left([0, T] ; L^{2}(\Omega)\right) \text { for } k+|\alpha| \leq m, l \in \mathbb{N} .
$$

and the compatibility condition of order $s+1$ :

$$
\partial_{t}^{k} f_{i}(z, 0)=0, \quad 0 \leq k \leq s
$$

Thanks to that the coefficient of $\partial_{z_{2}} u$ is vanished, we could obtain that

Proposition 4.3. Assume that the conditions (4.136) -(4.139) hold. Then there exists a unique solution to (4.134)-(4.135) satisfying

$$
\left\langle z_{1}\right\rangle^{l} \partial_{t}^{k} \partial_{z_{1}}^{\alpha_{1}} \partial_{z_{2}}^{\alpha_{2}} u_{i} \in C^{0}\left([0, T] ; L^{2}(\Omega)\right)
$$

for $k+|\alpha| \leq m-1, k+\left[\frac{\alpha_{1}+1}{2}\right] \leq s$, and

$$
\partial_{t}^{k} u_{i}(z, 0)=0, \quad k=0,1, \ldots, \min (s, m-1), \quad i=1,2
$$

In order to prove Proposition 4.3, we consider the following initial boundary value problem for a small parameter $\delta>0$

$$
a\left(z_{2}, t\right) \partial_{t} \mathbf{u}+b\left(z_{2}, t\right) z_{1} \partial_{z_{1}} \mathbf{u}+c\left(z_{2}, t\right) \mathbf{u}-\partial_{z_{1}}^{2} \mathbf{u}-\delta \partial_{z_{2}}^{2} \mathbf{u}=\mathbf{f}^{\delta}(z, t),
$$

$(z, t) \in \Omega \times[0, T]$, with the boundary condition

$$
\begin{gathered}
\mathbf{u}=0, \quad \text { on } \Gamma \times[0, T] \\
\mathbf{u}(z, 0)=0, \text { for } z \in \Omega .
\end{gathered}
$$

where we denote

$$
\begin{gathered}
\mathbf{u}=\left(u_{1}, u_{2}\right)^{\top}, \\
a\left(z_{2}, t\right)=\left(\begin{array}{cc}
a_{1}\left(z_{2}, t\right) & 0 \\
0 & a_{2}\left(z_{2}, t\right)
\end{array}\right), \\
b\left(z_{2}, t\right)=\left(\begin{array}{ll}
b_{11}\left(z_{2}, t\right) & b_{12}\left(z_{2}, t\right) \\
b_{21}\left(z_{2}, t\right) & b_{22}\left(z_{2}, t\right)
\end{array}\right) \\
c\left(z_{2}, t\right)=\left(\begin{array}{ll}
c_{11}\left(z_{2}, t\right) & c_{12}\left(z_{2}, t\right) \\
c_{21}\left(z_{2}, t\right) & c_{22}\left(z_{2}, t\right)
\end{array}\right)
\end{gathered}
$$


and $\mathbf{f}^{\delta}=\left(f_{1}^{\delta}, f_{2}^{\delta}\right)^{\top}$ which is smooth and satisfies

$$
\partial_{t}^{k} \mathbf{f}^{\delta}(z, 0)=0, \text { for } k=0,1, \ldots s
$$

We could have the following uniform energy estimates, Proposition (4.3) is its corollary with the standard theory for linear parabolic equations and approximate process $(\delta \rightarrow 0)$. In the rest part of this section, the generic constant $C$ depend only on $a, b, c$ and are independent of $\delta$.

Lemma 4.1. The solution $\boldsymbol{u}^{\delta}$ of (4.141)-(4.142) satisfies that for any $t \in[0, T]$,

$$
\begin{gathered}
\sum_{k+|\alpha| \leq m, k+\left[\frac{\alpha_{1}+1}{2}\right] \leq s}\left\|\left\langle z_{1}\right\rangle^{l} \partial_{t}^{k} \partial_{z_{1}}^{\alpha_{1}} \partial_{z_{2}}^{\alpha_{2}} \boldsymbol{u}^{\delta}\right\|^{2} \\
\leq C \sum_{k+|| \alpha \mid \leq m, k+\left[\frac{\alpha_{1}+1}{2}\right] \leq s} \int_{0}^{t}\left\|\left\langle z_{1}\right\rangle^{l+m} \partial_{t}^{k} \partial_{z_{1}}^{\alpha_{1}} \partial_{z_{2}}^{\alpha_{2}} \boldsymbol{f}^{\delta}(z, s)\right\|^{2} \mathrm{~d} s .
\end{gathered}
$$

Proof. For simplify, we shall omit the parameter $\delta$. First, we get the $L^{2}$ estimate of $\mathbf{u}$. Multiplying (4.141) by $\left\langle z_{1}\right\rangle^{2 l} \mathbf{u}$, and integrating by part, so we obtain that

$$
\begin{aligned}
& \frac{1}{2} \frac{\mathrm{d}}{\mathrm{d} t} \int_{\Omega} \sum_{i=1,2}\left\langle z_{1}\right\rangle^{2 l} a_{i}\left|u_{i}\right|^{2} \mathrm{~d} z-\frac{1}{2} \int_{\Omega} \sum_{i=1,2}\left\langle z_{1}\right\rangle^{2 l} \partial_{t}\left(a_{i}\right)\left|u_{i}\right|^{2} \mathrm{~d} z \\
& +\int_{\Omega} \sum_{i, j=1,2}\left\langle z_{1}\right\rangle^{2 l} b_{i j} z_{1} u_{j} \partial_{z_{1}} u_{i} \mathrm{~d} z+\int_{\Omega} \sum_{i, j=1,2}\left\langle z_{1}\right\rangle^{2 l} c_{i j} u_{j} u_{i} \mathrm{~d} z \\
& +\int_{\Omega} \sum_{i=1,2} \partial_{z_{1}}\left(\left\langle z_{1}\right\rangle^{2 l} u_{i}\right) \partial_{z_{1}} u_{i} \mathrm{~d} z+\int_{\Omega} \sum_{i=1,2} \delta\left\langle z_{1}\right\rangle^{2 l}\left|\partial_{z_{2}} u_{i}\right|^{2} \mathrm{~d} z \\
& =\int_{\Omega} \sum_{i=1,2}\left\langle z_{1}\right\rangle^{2 l} f_{i} u_{i} \mathrm{~d} z,
\end{aligned}
$$

Note that

$$
\begin{aligned}
& \int_{\Omega} \sum_{i, j=1,2}\left\langle z_{1}\right\rangle^{2 l} b_{i j} z_{1} u_{j} \partial_{z_{1}} u_{i} \mathrm{~d} z \\
= & \frac{1}{2} \int_{\Omega} \sum_{i, j=1,2} b_{i j} \partial_{z_{1}}\left(\left\langle z_{1}\right\rangle^{2 l} z_{1} u_{i} u_{j}\right) d z-\frac{1}{2} \int_{\Omega} \sum_{i, j=1,2} b_{i j} \partial_{z_{1}}\left(\left\langle z_{1}\right\rangle^{2 l} z_{1}\right) u_{i} u_{j} \mathrm{~d} z \\
= & -\frac{1}{2} \int_{\Omega} \sum_{i, j=1,2} b_{i j} \partial_{z_{1}}\left(\left\langle z_{1}\right\rangle^{2 l} z_{1}\right) u_{i} u_{j} \mathrm{~d} z
\end{aligned}
$$

Then we could have that for any $t \in[0, T]$,

$$
\begin{array}{r}
\sum_{i=1,2}\left\|\left\langle z_{1}\right\rangle^{l} u_{i}(t)\right\|^{2}+\int_{0}^{t} \sum_{i=1,2}\left\|\left\langle z_{1}\right\rangle^{l} \partial_{z_{1}} u_{i}(s)\right\|^{2} \mathrm{~d} s \\
+\delta \int_{0}^{t} \sum_{i=1,2}\left\|\left\langle z_{1}\right\rangle^{l} \partial_{z_{2}} u_{i}(s)\right\|^{2} \mathrm{~d} s \leq C \int_{0}^{t}\left\|\left\langle z_{1}\right\rangle^{l} \mathbf{f}^{\delta}(s)\right\|^{2} \mathrm{~d} s .
\end{array}
$$


Next, we estimate the derivative with respect to $z_{2}$. Applying $\partial_{z_{2}}^{\alpha_{2}}$ to (4.141), multiplying these equations by $\left\langle z_{1}\right\rangle^{2 l} \partial_{z_{2}}^{\alpha_{2}} \mathbf{u}$, we have that

$$
\begin{aligned}
& \frac{1}{2} \frac{\mathrm{d}}{\mathrm{d} t} \int_{\Omega} \sum_{i=1,2}\left\langle z_{1}\right\rangle^{2 l} a_{i}\left|\partial_{z_{2}}^{\alpha_{2}} u_{i}\right|^{2} \mathrm{~d} z-\frac{1}{2} \int_{\Omega} \sum_{i=1,2}\left\langle z_{1}\right\rangle^{2 l} \partial_{t}\left(a_{i}\right)\left|\partial_{z_{2}}^{\alpha_{2}} u_{i}\right|^{2} \mathrm{~d} z \\
& -\int_{\Omega} \sum_{i, j=1,2} \partial_{z_{1}}\left(\left\langle z_{1}\right\rangle^{2 l} z_{1}\right) b_{i j} \partial_{z_{2}}^{\alpha_{2}} u_{j} \partial_{z_{2}}^{\alpha_{2}} u_{i} \mathrm{~d} z+\int_{\Omega} \sum_{i, j=1,2}\left\langle z_{1}\right\rangle^{2 l} c_{i j} \partial_{z_{2}}^{\alpha_{2}} u_{j} \partial_{z_{2}}^{\alpha_{2}} u_{i} \mathrm{~d} z \\
& \quad+\int_{\Omega} \sum_{i=1,2} \partial_{z_{1}}\left(\left\langle z_{1}\right\rangle^{2 l} \partial_{z_{2}}^{\alpha_{2}} u_{i}\right) \partial_{z_{1}} \partial_{z_{2}}^{\alpha_{2}} u_{i} \mathrm{~d} z+\int_{\Omega} \sum_{i=1,2} \delta\left\langle z_{1}\right\rangle^{2 l}\left|\partial_{z_{2}} \partial_{z_{2}}^{\alpha_{2}} u_{i}\right|^{2} \mathrm{~d} z \\
& =\int_{\Omega} \sum_{i=1,2}\left\langle z_{1}\right\rangle^{2 l} \partial_{z_{2}}^{\alpha_{2}} f_{i}^{\delta} \partial_{z_{2}}^{\alpha_{2}} u_{i} \mathrm{~d} z-\int_{\Omega} \sum_{i=1,2}\left\langle z_{1}\right\rangle^{2 l}\left[\partial_{z_{2}}^{\alpha_{2}}, a_{i}\right] \partial_{t} u_{i} \partial_{z_{2}}^{\alpha_{2}} u_{i} \mathrm{~d} z \\
& \quad-\int_{\Omega} \sum_{i, j=1,2}\left\langle z_{1}\right\rangle^{2 l}\left[\partial_{z_{2}}^{\alpha_{2}}, b_{i j}\right] z_{1} \partial_{z_{1}} u_{i} \partial_{z_{2}}^{\alpha_{2}} u_{j} \mathrm{~d} z \\
& -\int_{\Omega} \sum_{i, j=1,2}\left\langle z_{1}\right\rangle^{2 l}\left[\partial_{z_{2}}^{\alpha_{2}}, c_{i j}\right] u_{i} \partial_{z_{2}}^{\alpha_{2}} u_{j} \mathrm{~d} z
\end{aligned}
$$

For the main difficult term $\int_{\Omega} \sum_{i=1,2}\left\langle z_{1}\right\rangle^{2 l}\left[\partial_{z_{2}}^{\alpha_{2}}, a_{i}\right] \partial_{t} u_{i} \partial_{z_{2}}^{\alpha_{2}} u_{i}$, we have that

$$
\begin{aligned}
& \int_{\Omega} \sum_{i=1,2}\left\langle z_{1}\right\rangle^{2 l}\left[\partial_{z_{2}}^{\alpha_{2}}, a_{i}\right] \partial_{t} u_{i} \partial_{z_{2}}^{\alpha_{2}} u_{i} \\
= & -\int_{\Omega} \sum_{i=1,2}\left\langle z_{1}\right\rangle^{2 l}\left[\partial_{z_{2}}^{\alpha_{2}}, a_{i}\right] \frac{1}{a_{i}}\left\{\sum_{j=1,2}\left(b_{i j} z_{1} \partial_{z_{1}} u_{j}+c_{i j} u_{j}\right)\right. \\
& \left.-\partial_{z_{1}}^{2} u_{i}-\delta \partial_{z_{2}}^{2} u_{i}-f_{i}^{\delta}\right\} \partial_{z_{2}}^{\alpha_{2}} u_{i},
\end{aligned}
$$

and

$$
\begin{aligned}
& \left|\int_{\Omega} \sum_{i=1,2}\left\langle z_{1}\right\rangle^{2 l}\left[\partial_{z_{2}}^{\alpha_{2}}, a_{i}\right] \frac{1}{a_{i}} \partial_{z_{1}}^{2} u_{i} \partial_{z_{2}}^{\alpha_{2}} u_{i} \mathrm{~d} z\right| \\
= & \mid-\int_{\Omega} \sum_{i=1,2} \partial_{z_{1}}\left(\left[\partial_{z_{2}}^{\alpha_{2}}, a_{i}\right]\right) \frac{1}{a_{i}} \partial_{z_{1}} u_{i}\left\langle z_{1}\right\rangle^{2 l} \partial_{z_{2}}^{\alpha_{2}} u_{i} \mathrm{~d} z- \\
& \int_{\Omega} \sum_{i=1,2}\left[\partial_{z_{2}}^{\alpha_{2}}, a_{i}\right] \frac{1}{a_{i}} \partial_{z_{1}} u_{i} \partial_{z_{1}}\left(\left\langle z_{1}\right\rangle^{2 l} \partial_{z_{2}}^{\alpha_{2}} u_{i}\right) \mathrm{d} z \mid \\
\leq & C \sum_{j \leq \alpha_{2}-1}\left\|\left\langle z_{1}\right\rangle^{l} \partial_{z_{1}} \partial_{z_{2}}^{j} u_{i}\right\|^{2}+\frac{1}{4}\left\|\left\langle z_{1}\right\rangle^{l} \partial_{z_{1}} \partial_{z_{2}}^{\alpha_{2}} u_{i}\right\|^{2}+\left\|\left\langle z_{1}\right\rangle^{l} \partial_{z_{2}}^{\alpha_{2}} u_{i}\right\|^{2},
\end{aligned}
$$

Then from (4.147), we could get that

$$
\begin{aligned}
& \sum_{\alpha_{2}=1}^{m}\left\{\left\|\left\langle z_{1}\right\rangle^{l} \partial_{z_{2}}^{\alpha_{2}} \mathbf{u}(t)\right\|^{2}+\int_{0}^{t}\left\|\left\langle z_{1}\right\rangle^{l} \partial_{z_{1}} \partial_{z_{2}}^{\alpha_{2}} \mathbf{u}(s)\right\|^{2} \mathrm{~d} s\right\} \\
\leq & C \sum_{\alpha_{2}=0}^{m} \int_{0}^{t}\left\|\left\langle z_{1}\right\rangle^{l} \partial_{z_{2}}^{\alpha_{2}} \mathbf{f}^{\delta}(s)\right\|^{2} \mathrm{~d} s .
\end{aligned}
$$

Applying $\partial_{t}^{k}$ to the equation (4.141), similarly, one could get that 


$$
\begin{gathered}
\left.\sup _{t \in[0, T]}\left\|\left\langle z_{1}\right\rangle^{l} \partial_{t}^{k} \mathbf{u}(t)\right\|^{2}+\int_{0}^{t}\left\|\left\langle z_{1}\right\rangle^{l} \partial_{z_{1}} \partial_{t}^{k} \mathbf{u}(s)\right\|^{2} \mathrm{~d} s\right\} \\
\leq C\left\{\int_{0}^{\top}\left\|\left\langle z_{1}\right\rangle^{l} \partial_{t}^{k} \mathbf{f}^{\delta}(s)\right\|^{2} \mathrm{~d} s+\sum_{k^{\prime} \leq k-1}\left[T \operatorname { s u p } _ { t \in [ 0 , T ] } \left(\left\|\left\langle z_{1}\right\rangle^{l} \partial_{t}^{k^{\prime}} \mathbf{u}(t)\right\|^{2}\right.\right.\right. \\
\left.\left.\left.+\left\|\left\langle z_{1}\right\rangle^{l} \partial_{z_{2}} \partial_{t}^{k^{\prime}} \mathbf{u}(t)\right\|^{2}\right)+\int_{0}^{\top}\left\|\left\langle z_{1}\right\rangle^{l} \partial_{z_{1}} \partial_{t}^{k^{\prime}} \mathbf{u}(s)\right\|^{2} \mathrm{~d} s\right]\right\} .
\end{gathered}
$$

Applying $\partial_{z_{2}}^{\alpha_{2}} \partial_{t}^{k}$ to the equation (4.141), and using (4.151), similar as the estimate (4.150), we have that

$$
\begin{aligned}
& \sum_{k+\alpha_{2} \leq m, 0 \leq k \leq s}\left\|\left\langle z_{1}\right\rangle^{l} \partial_{t}^{k} \partial_{z_{2}}^{\alpha_{2}} \mathbf{u}^{\delta}\right\|^{2}+\sum_{k+\alpha_{2} \leq m, 0 \leq k \leq s} \int_{0}^{t}\left\|\left\langle z_{1}\right\rangle^{l} \partial_{t}^{k} \partial_{z_{2}}^{\alpha_{2}} \mathbf{u}(s)\right\|^{2} \mathrm{~d} s \\
\leq C & \sum_{k+\alpha_{2} \leq m, 0 \leq k \leq s} \int_{0}^{t}\left\|\left\langle z_{1}\right\rangle^{l+m} \partial_{t}^{k} \partial_{z_{2}}^{\alpha_{2}} \mathbf{f}^{\delta}(z, s)\right\|^{2} \mathrm{~d} s .
\end{aligned}
$$

Finally, we could estimate the normal derivative. From (4.152), we have

$$
\begin{gathered}
\sum_{k+\alpha_{2} \leq m-1,0 \leq k \leq s-1}\left\|\left\langle z_{1}\right\rangle^{l} \partial_{z_{1}} \partial_{t}^{k} \partial_{z_{2}}^{\alpha_{2}} \mathbf{u}(t)\right\|^{2} \\
\leq C \sum_{k+\alpha_{2} \leq m, 0 \leq k \leq s} \int_{0}^{t}\left\|\left\langle z_{1}\right\rangle^{l+m} \partial_{t}^{k} \partial_{z_{2}}^{\alpha_{2}} \mathbf{f}^{\delta}(z, s)\right\|^{2} \mathrm{~d} s .
\end{gathered}
$$

Note that $\partial_{z_{1}}^{2}=a\left(z_{2}, t\right) \partial_{t} \mathbf{u}+b\left(z_{2}, t\right) z_{1} \partial_{z_{1}} \mathbf{u}+c\left(z_{2}, t\right) \mathbf{u}--\delta \partial_{z_{2}}^{2} \mathbf{u}-\mathbf{f}^{\delta}(z, t)$, iteratively, we could get that

$$
\begin{gathered}
\sum_{k+|\alpha| \leq m, k+\left[\frac{\alpha_{1}+1}{2}\right] \leq s}\left\|\left\langle z_{1}\right\rangle^{l} \partial_{t}^{k} \partial_{z_{1}}^{\alpha_{1}} \partial_{z_{2}}^{\alpha_{2}} \mathbf{u}^{\delta}\right\|^{2} \\
\leq C \sum_{k+|| \alpha \mid \leq m, k+\left[\frac{\alpha_{1}+1}{2}\right] \leq s} \int_{0}^{t}\left\|\left\langle z_{1}\right\rangle^{l+m} \partial_{t}^{k} \partial_{z_{1}}^{\alpha_{1}} \partial_{z_{2}}^{\alpha_{2}} \mathbf{f}^{\delta}(z, s)\right\|^{2} \mathrm{~d} s .
\end{gathered}
$$

The proof of Lemma 4.1 is complete.

Acknowledgment: Ning Jiang was supported by a grant from the National Natural Science Foundation of China under contract No. 11171173. Yutao Ding was supported by the postdoctoral funding of Mathematical Science Center of Tsinghua University. Ning Jiang also appreciate Prof. Z.P.Xin for his invitation of the visit to the Institute of Mathematical Sciences of CUHK between Feb-April 2012. During the visit, the conversation and suggestion of Prof. Xin play an important role in this work.

\section{REFERENCES}

[1] E. Feireisl, Dynamics of viscous compressible fluids. Oxford Lecture Series in Mathematics and its Applications, 26. Oxford University Press, Oxford, 2004.

[2] N. Jiang and N. Masmoudi, The acoustic dynamics of the linearized Boltzmann equation in half space with Maxwell reflection boundary condition. (In preparation).

[3] A. Matsumura and T. Nishida, Initial-boundary value problems for the equations of motion of compressible viscous and heat-conductive fluids. Comm. Math. Phys. 89 (1983), no. 4, 445-464.

[4] M. Sammartino,and R. Caflisch, Zero viscosity limit for analytic solutions of the Navier-Stokes equation on a half-space. I. Existence for Euler and Prandtl equations. Comm. Math. Phys. 192, 2 (1998), 433-461.

[5] M. Sammartino,and R. Caflisch, Zero viscosity limit for analytic solutions of the Navier-Stokes equation on a half-space. II. Existence for Euler and Prandtl equations. Comm. Math. Phys. 192, 2 (1998), 463-491. 
[6] S. Schochet, The compressible Euler equations in a bounded domain: existence of solutions and the incompressible limit. Comm. Math. Phys. 104 (1986), no.1, 49-75.

[7] Z. Xin, Viscous boundary layers and their stability. I. J. Partial Differential Equations. 11 (1998), no. 2, 97-124.

[8] Z. Xin and T. Yanagisawa, Zero-viscosity limit of the linearized Navier-Stokes Equations for a Compressible viscous fluid in the half-plane. Comm. Pure Appl. Math. 52 (1999), no. 4, 479-541

[9] Z. Xin, and L. Zhang, On the global existence of solutions to the Prandtl's system. Adv. Math. 181, 1 (2004), 88-133

Mathematical Sciences Center, Jin Chunyuan West Building, Beijing, 100084

E-mail address: ytding@math.tsinghua.edu.cn

Mathematical Sciences Center, Jin Chunyuan West Building, Beijing, 100084

E-mail address: njiang@math.tsinghua.edu.cn 